\title{
Differential Neurotoxicity Related to Tetracycline Transactivator and TDP-43 Expression in Conditional TDP-43 Mouse Model of Frontotemporal Lobar Degeneration
}

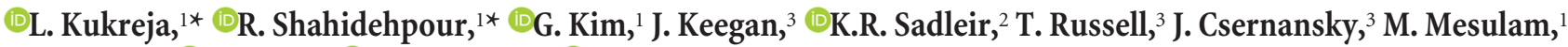 \\ R.J. Vassar, ${ }^{\circ}$ L. Wang, ${ }^{3} \odot$ H. Dong, ${ }^{3}$ and $\oplus^{\circ}$. Geula ${ }^{1,2}$ \\ ${ }^{1}$ Cognitive Neurology and Alzheimer's Disease Center, ${ }^{2}$ Department of Cell and Molecular Biology, and ${ }^{3}$ Department of Psychiatry and Behavioral Science, \\ Northwestern University, Feinberg School of Medicine, Chicago, Illinois 60626
}

Frontotemporal lobar degeneration (FTLD) is among the most prevalent dementias of early-onset. Pathologically, FTLD presents with tauopathy or TAR DNA-binding protein 43 (TDP-43) proteinopathy. A biallelic mouse model of FTLD was produced on a mix FVB/ 129SVE background overexpressing wild-type human TDP-43 (hTDP-43) using tetracycline transactivator (tTA), a system widely used in mouse models of neurological disorders. tTA activates hTDP-43, which is placed downstream of the tetracycline response element. The original study on this transgenic mouse found hippocampal degeneration following hTDP-43 expression, but did not account for independent effects of tTA protein. Here, we initially analyzed the neurotoxic effects of tTA in postweaning age mice of either sex using immunostaining and area measurements of select brain regions. We observed tTA-dependent toxicity selectively in the hippocampus affecting the dentate gyrus significantly more than CA fields, whereas hTDP-43-dependent toxicity in bigenic mice occurred in most other cortical regions. Atrophy was associated with inflammation, activation of caspase-3, and loss of neurons. The atrophy associated with tTA expression was rescuable by the tetracycline analog, doxycycline, in the diet. MRI studies corroborated the patterns of atrophy. tTAinduced degeneration was strain-dependent and was rescued by moving the transgene onto a congenic C57BL/6 background. Despite significant hippocampal atrophy, behavioral tests in bigenic mice revealed no hippocampally mediated memory impairment. Significant atrophy in most cortical areas due solely to TDP- 43 expression indicates that this mouse model remains useful for providing critical insight into co-occurrence of TDP-43 pathology, neurodegeneration, and behavioral deficits in FTLD.

Key words: conditional transgenic; doxycycline transducer toxicity; frontotemporal dementia; frontotemporal lobar degeneration; TDP-43 toxicity

Significance Statement

The tTA expression system has been widely used in mice to model neurological disorders. The technique allows investigators to reversibly turn on or off disease causing genes. Here, we report on a mouse model that overexpresses human TDP-43 using tTA and attempt to recapitulate features of TDP-43 pathology present in human FTLD. The tTA expression system is problematic, resulting in dramatic degeneration of the hippocampus. Thus, our study adds a note of caution for the use of the tTA system. However, because FTLD is primarily characterized by cortical degeneration and our mouse model shows significant atrophy in most cortical areas due to human TDP-43 overexpression, our animal model remains useful for providing critical insight on this human disease.

\section{Introduction}

Dementias caused by frontotemporal lobar degeneration (FTLD) constitute the third most prevalent dementia, after those caused by Alzheimer's disease and Lewy bodies, and are among the most

Received June 28, 2017; revised April 23, 2018; accepted May 1, 2018.

Author contributions: L.K., J.C., M.M., R.J.V., L.W., H.D., and C.G. designed research; L.K., R.S., G.K., J.K., K.R.S., T.R., L.W., H.D., and C.G. performed research; L.K., R.S., L.W., H.D., and C.G. analyzed data; L.K., R.S., and C.G. wrote the paper.

*L.K. and R.S. contributed equally to this work. prevalent dementias of early-onset (Cairns et al., 2007). The majority of cases are characterized by behavioral and emotional

This work was supported by National Institute of Neurological Disorder and Stroke (NS085770) and the Lewis Foundation. The conditional transgenic mouse model of FTLD was a generous gift from Dr. Jada Lewis (University of Florida (enter for Translational Research in Neurodegenerative Disease).

The authors declare no competing financial interests.

Correspondence should be addressed to Dr. Changiz Geula, Cognitive Neurology and Alzheimer's Disease Center, Northwestern University, Feinberg School of Medicine, Searle 11-467, Chicago, IL 60611. E-mail: c-geula@northwestern.edu. DOl:10.1523/JNEUROSCI.1836-17.2018

Copyright $\odot 2018$ the authors $\quad 0270-6474 / 18 / 386045-18 \$ 15.00 / 0$ 
A

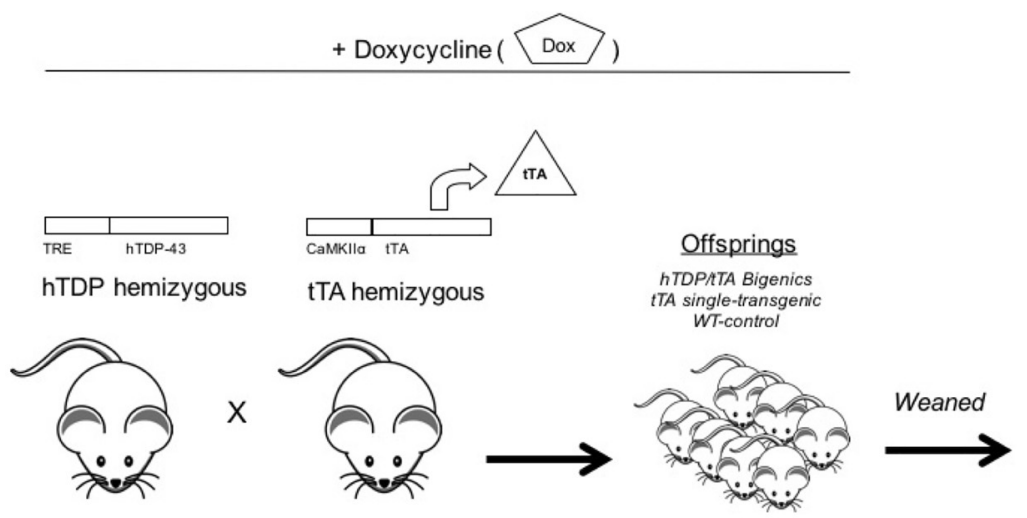

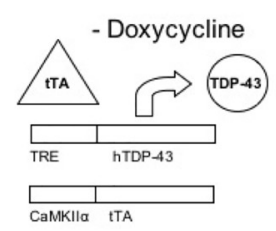

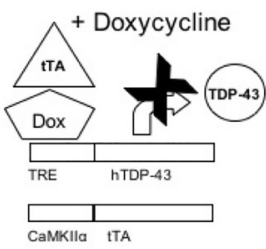

Aged $8 \& 24$ wks
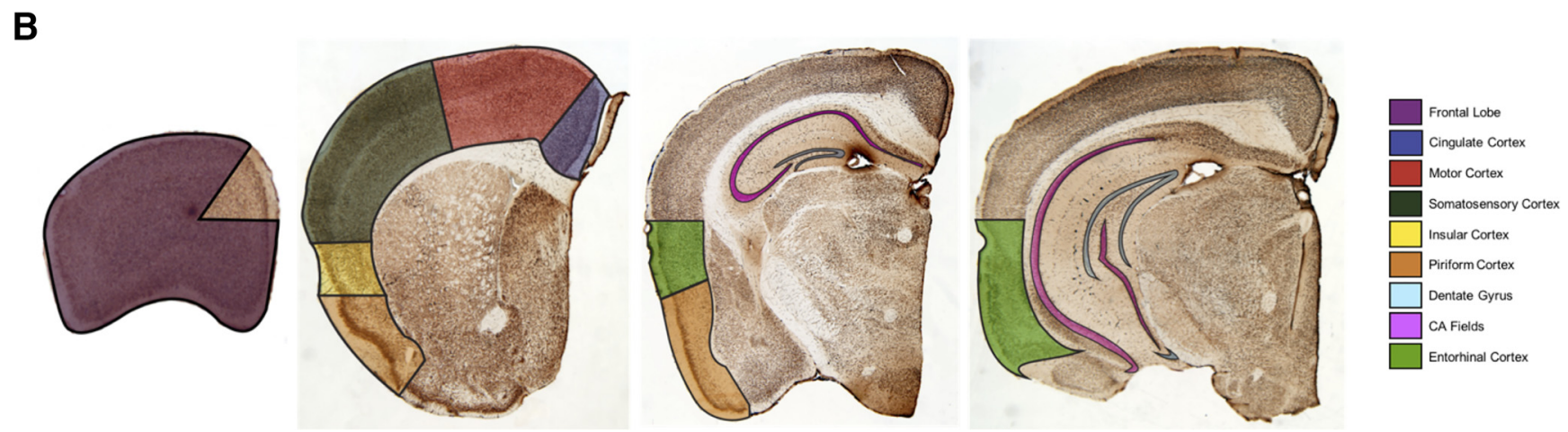

Figure 1. Overexpression of wild-type hTDP-43 in a conditional transgenic mouse. A, Mice overexpressing wild-type hTDP-43 were produced by crossing hemizygous hTDP-43-expressing mice with hemizygous tTA transgenic animals. The full-length hTDP-43 CDNA was placed behind a minimal CMV promoter, which contained five TREs, effectively blocking expression in hemizygous hTDP-43 transgenic mice. By crossing these mice with mice expressing the TTA under CaMKIla, tTA/hTDP-43 bigenic mice were created, which subsequently expressed the hTDP-43 protein specifically in the excitatory neurons of the forebrain. The cross also produced wild-type, and tTA single transgenic mice. Administration of dox in animal diet blocks tTA from binding to TRE and prevents $\mathrm{hTDP}-43$ expression. All animals were kept on dox diet until weaning age and then either came off dox diet, which would allow $\mathrm{tTA} / \mathrm{hTDP}-43$ bigenic mice to express the $\mathrm{hTDP}-43$ transgene, or remained on dox for another 8 or 24 weeks. $\boldsymbol{B}$, Sections cut at $40 \mu \mathrm{m}$ were selected for examination based on their distance from bregma $(2.58,0.86,-2.54$, and $-3.40 \mathrm{~mm}$; Paxinos and Franklin, 2001). Sections analyzed contained the following anatomical structures: dorsolateral prefrontal cortex, anterior cingulate cortex, motor cortex, somatosensory cortex, insula, piriform olfactory cortex, granular layer of the DG, the CA fields of the hippocampus, and the entorhinal cortex.

disturbances, primarily disinhibition and/or apathy. This phenotype is known as the behavioral variant frontotemporal dementia (bvFTD; Piguet et al., 2011). Anatomically, the brains of bvFTD patients present with atrophy in the prefrontal cortex and the anterior temporal lobes (Cairns et al., 2007).

In the majority of bvFTD patients, abnormal precipitates of phosphorylated and mislocalized TAR DNA-binding protein 43 (TDP-43) deposit in the brain in the form of inclusions, designated FTLD-TDP (Cairns et al., 2007; Kwong et al., 2007). The mislocalization of TDP-43 from the nucleus to the cytoplasm is an important step in TDP-43 aggregation and potential toxicity (Barmada et al., 2010).

TDP-43 is a 414 aa protein with two RNA recognition motifs, which displays a strictly nuclear localization in normal brains (Chen-Plotkin et al., 2010; Lee et al., 2011), where it binds a large number of RNA species $(>6000)$ and thereby controls RNA transcription, splicing and transport, and levels of microRNAs (Buratti et al., 2010; Tollervey et al., 2011; Narayanan et al., 2013). Several mouse models have shown that overexpression of wildtype or mutant human TDP-43 gene results in the formation of inclusions and neuronal loss, indicating that TDP-43 pathology contributes to FTLD (Xu et al., 2010; Cannon et al., 2012; D'alton et al., 2014).
Here we report on a mouse model of FTLD that overexpresses the wild-type human TDP-43 (hTDP-43) gene using the tetracycline transactivator (tTA), a system widely used to model neurological disorders. Transgenic expression of tTA was used to activate the wild-type human TDP-43 gene, which was placed downstream of the tetracycline response element (TRE). Exposure to doxycycline (dox), a more stable analog of tetracycline, causes a conformational change in tTA that inhibits binding to TRE and stops the expression of the TRE-controlled transgene. This allowed us to control when hTDP-43 expression is turned on. A conditional transgenic system was necessary for investigation of TDP-43 pathology because TDP-43 has important developmental functions. TDP-43 overexpression during early postnatal life has been determined to lead to premature mortality (Xu et al., 2010; Cannon et al., 2012), requiring that expression remain turned off until after weaning age. Although the biallelic system of transgene and tTA is powerful, there are caveats in its use.

A recent study found that tTA exhibits its own toxicity that is influenced by the genetic background strain of mice (Han et al., 2012). Granule neurons of the dentate gyrus (DG) appeared most vulnerable (Han et al., 2012; Liu et al., 2015). Our tTA and hTDP-43 transgenic mice were bred on 129SVE and FVB backgrounds, respectively, which are among the few mouse strains 


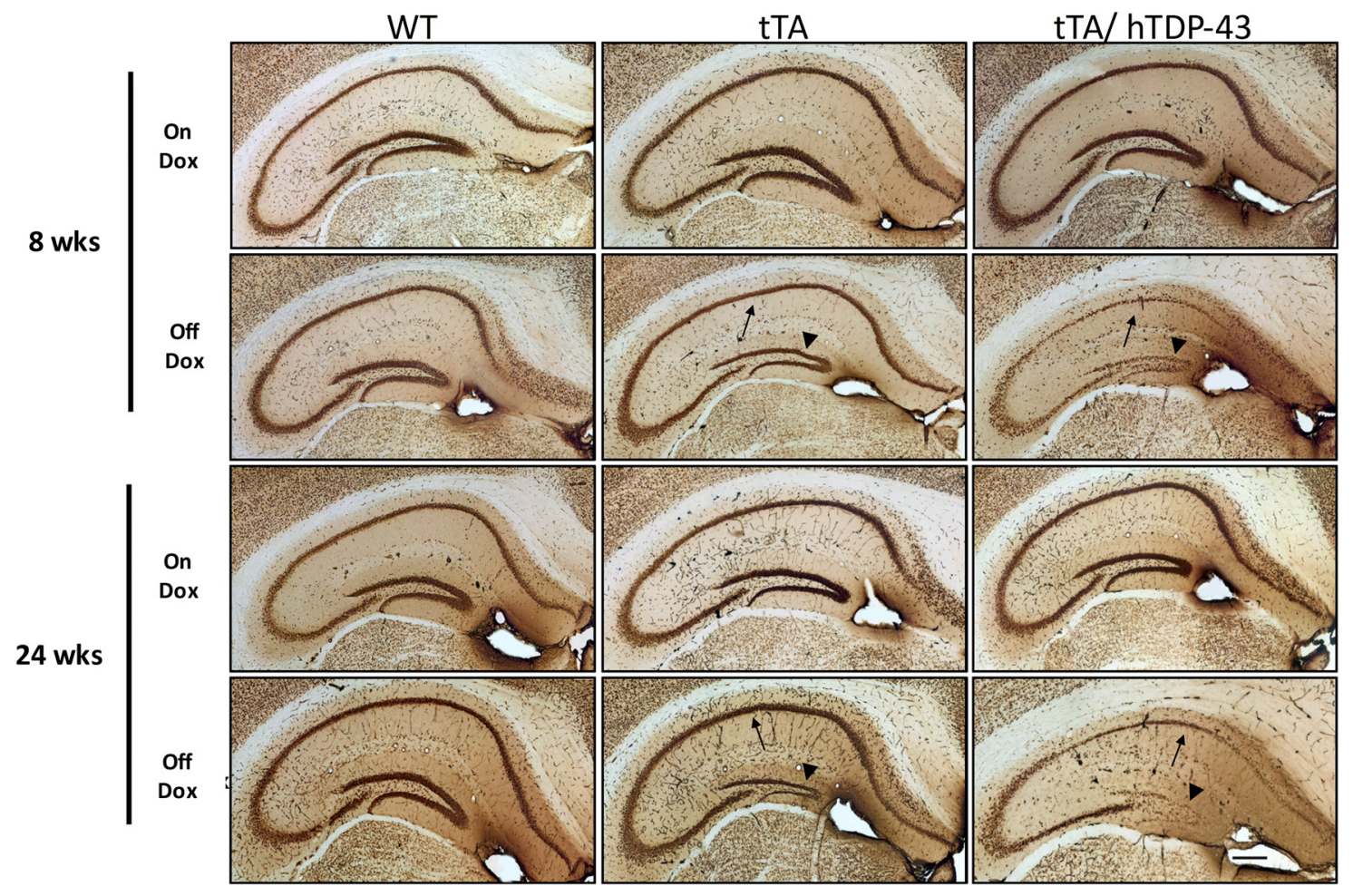

Figure 2. Similar pattern of hippocampal degeneration is observed in single tTA transgenic and tTA/hTDP-43 bigenic mice. Representative sections cut at $40 \mu \mathrm{m}$ and stained for NeuN show significant degeneration of the granular layer of the DG (arrowheads) and the CA fields of the hippocampus (arrows) in tTA single transgenic and bigenic animals (TTA/hTDP-43) off dox at 8 and 24 weeks postweaning age compared with WT mice. Loss of granular cells in DG is evident. Degeneration due to tTA and hTDP-43 expression is ameliorated with the addition of dox to the diet. Scale bar, $200 \mu \mathrm{m}$.

susceptible to neurodegeneration from tTA. Here we report on brain atrophy in mice at 8 - and 24 -week postweaning ages related to tTA expression. tTA toxicity, as measured by cortical atrophy, appeared to be concentrated in the mid-hippocampal region, and was dependent on the background strain of the mouse. First, the DG exhibited significant degeneration of granule cells. The tTA toxicity also extended to the cornu ammonis (CA) regions. Interestingly, this toxicity did not lead to deficits in hippocampal dependent reference memory measured by the Morris water maze. The atrophy associated with tTA was accompanied by inflammation, activation of caspase- 3 and loss of neurons. hTDP-43 exerted its own toxicity in selective cortical regions, independent of tTA expression. Our findings of cortical atrophy were corroborated by MR imaging results. Although this study adds a note of caution for using the tTA system to generate transgenic mice, our model remains useful for providing insight into TDP-43-specific pathology coinciding with neocortical neuron loss and behavioral deficits seen clinically in FTLD.

\section{Materials and Methods}

Animals. The inducible hTDP-43 transgenic mice were generously provided by Dr. Jada Lewis (Department of Neuroscience, University of Florida; Cannon et al., 2012). This model uses a biallelic system of responder (hTDP-43) and effector (tTA) transgenes. In this tetracyclineresponsive regulatory system, $\mathrm{tTA}$ is used to activate a second transgene of interest, i.e., the wild-type hTDP-43, which is placed downstream of the tetracycline response element (TRE). Exposure to dox, a more stable analog of tetracycline, in mouse diet causes a conformational change in tTA and inhibits its binding to TRE to then stop the expression of hTDP-43 transgene. By crossing TDP-43 WT mice with mice expressing tTA under the calcium calmodulin kinase II $\alpha$ promoter (CaMKIIa), tTA/hTDP-43 bigenic mice can be generated that express the hTDP-43 protein only in the excitatory neurons of the forebrain.
The original homozygous TDP-43 responder on the FVB mouse strain background were bred with wild-type FVB mice (Charles River Laboratories, IMSR Catalog \#CRL:207; RRID:IMSR_CRL:207) to maintain the responder colony and produce hemizygous TDP-43 animals. The homozygous tTA effector on the 129SVE mouse strain background were bred with wild-type 129SVE mice (Taconic, IMSR Catalog \#TAC:129sve; RRID:IMSR_TAC:129sve) to maintain the effector colony and produce hemizygous tTA mice.

Mice hemizygous for TDP-43 allele on FVB background and hemizygous for tTA allele on 129SVE background were then crossbred under dox treatment to produce monogenic and bigenic offspring of these two alleles with mix FVB and 129SVE background. Offspring of either sex from this cross that were used for the study included (1) hemizygous TDP43; hemizygous tTA (tTA/hTDP-43 bigenic), (2) hemizygous tTA (tTA single-transgenic), and (3) wild-type (WT-control).

This expression system allowed us to turn the TDP-43 transgene on and off at varying times to explore the effect of transgene expression at different ages (Gossen and Bujard, 1992; Cannon et al., 2012). Up until weaning age, all offspring were kept on dox diet. Then, animals were raised under 12 different conditions; for each genotype (WT, tTA, or tTA/hTDP-43), animals were aged either on dox or off dox diet for 8 or 24 weeks postweaning age (Fig. $1 A$ ).

After 8 or 24 weeks of transgene expression postweaning age, animals were deeply anesthetized with $100 \mathrm{mg} / \mathrm{kg}$ ketamine $/ 10 \mathrm{mg} / \mathrm{kg}$ xylazine in PBS $(0.1 \mathrm{ml} / 20$ g, i.p.). Mice were then perfused transcardially with phosphate buffer with a mixture of protease and phosphatase inhibitors. After perfusion, brains were removed, and one hemisphere was drop-fixed in $4 \%$ paraformaldehyde in $\mathrm{PBS}$ overnight at $4^{\circ} \mathrm{C}$ followed by incubation in $20 \%$ sucrose in PBS with $0.01 \%$ sodium azide at $4^{\circ} \mathrm{C}$ for at least $12 \mathrm{~h}$.

In addition to the mice on FVB and 129SVE mix background, the effect of tTA transgene expression was also analyzed on C57BL/6 mouse background. These mice were acquired from The Jackson Laboratory (strain 3010, IMSR Catalog \#JAX003010; RRID:IMSR_JAX:003010) and aged to 8 or 24 weeks postweaning. 
A Dentate Gyrus
Bregma $-2.54 \mathrm{~mm}(8$ weeks)
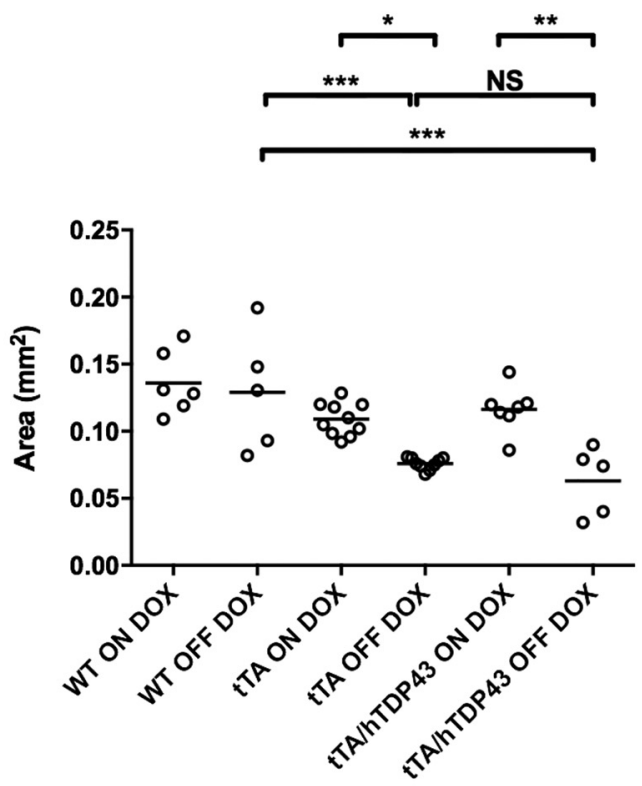

B

$$
\begin{gathered}
\text { Dentate Gyrus } \\
\text { Bregma }-3.40 \mathrm{~mm} \text { (8 weeks) }
\end{gathered}
$$
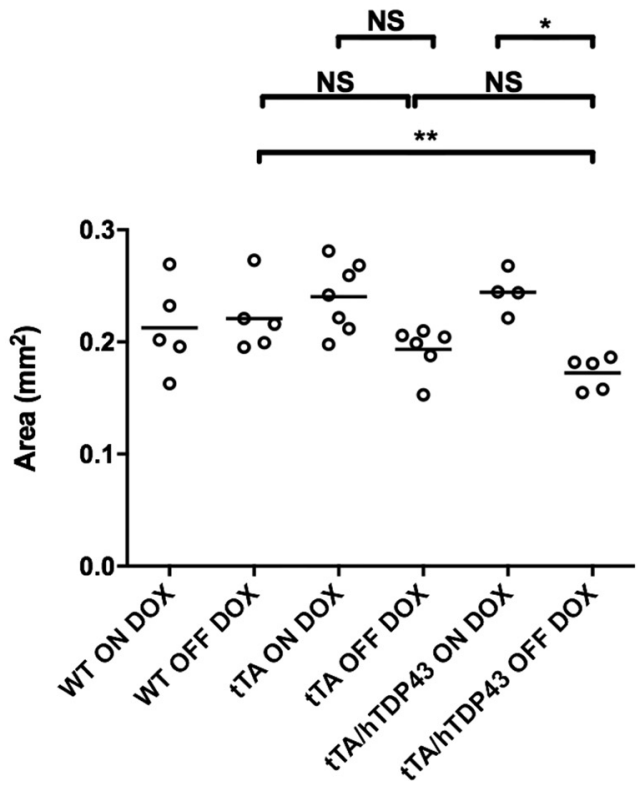

\section{Dentate Gyrus \\ Bregma -2.54 mm (24 weeks)}
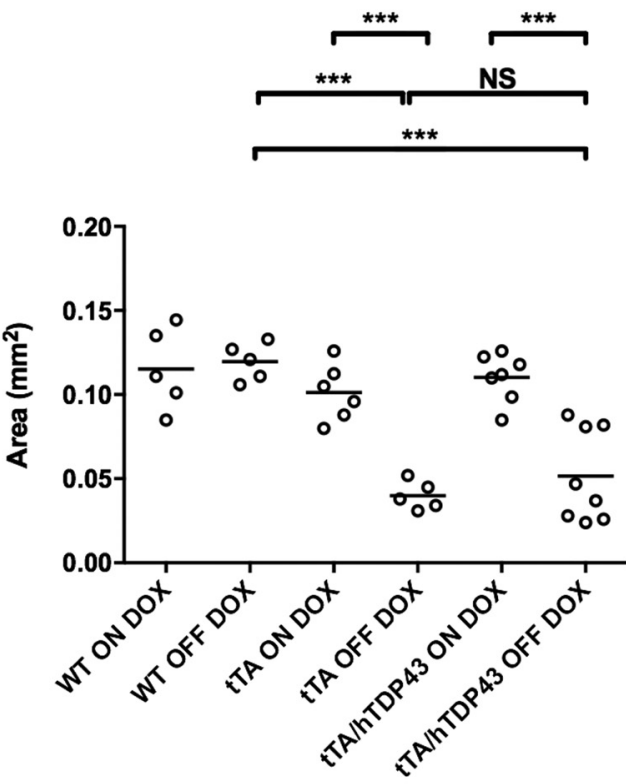

\section{Dentate Gyrus \\ Bregma $-3.40 \mathrm{~mm}(24$ weeks $)$}
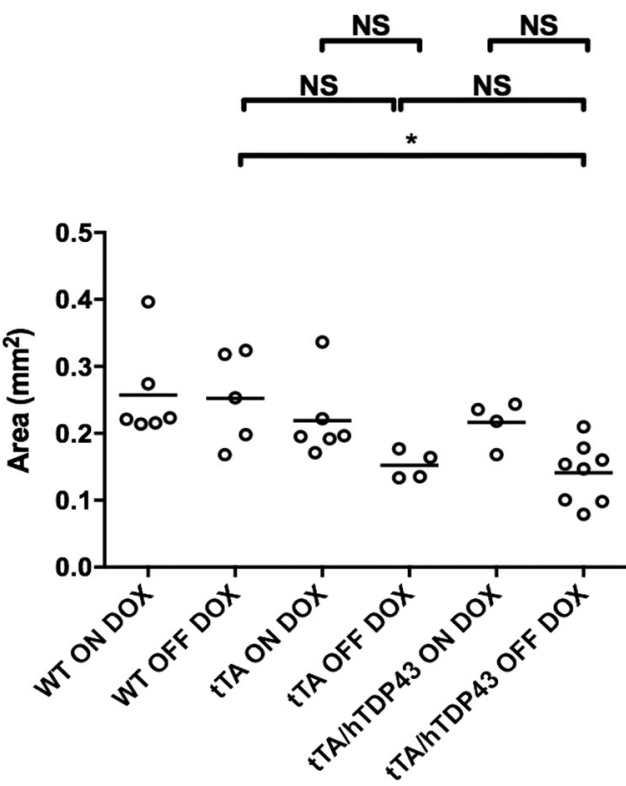

Figure 3. Quantitative analysis of degeneration in the dentate gyrus. A, Mice expressing only the tTA transgene off dox show significant atrophy in the DG in the mid-hippocampal subregion (represented in bregma $-2.54 \mathrm{~mm}$ ), which spreads to posterior hippocampus (represented in bregma $-3.40 \mathrm{~mm}$ ) at 8 weeks postweaning age. tTA/hTDP-43 bigenic mice off dox display an identical pattern of atrophy at 8 weeks to tTA single transgenic off dox mice. At 24 weeks postweaning age, tTA and tTA/hTDP-43 transgenic mice show increased levels of atrophy compared with WT mice. However, tTA/hTDP-43 bigenic mice show no significant difference compared with tTA transgenic mice. Treatment with dox ameliorated the atrophy in DG in these cohorts of mice. $B$, Posterior regions of the DG in bigenic mice off dox show smaller but similar patterns of atrophy compared with the mid-hippocampal region. $n=4-8$ mice for all genotypes; circles represent individual data points, horizontal lines represent the mean. ${ }^{*} p=0.027-0.011,{ }^{* *} p \leq 0.01,{ }^{* * *} p \leq 0.001$; one-way ANOVA followed by Bonferroni's multiple-comparison test post hoc.

To confirm animal genotypes, PCR was conducted using amplification of tail-clip DNA. Touchdown PCR was performed on a cycling program where the annealing temperature is gradually reduced. tTA and hTDP-43 alleles were amplified using PCR.
Mice were maintained in micro-isolator cages in barrier facilities. All animal procedures were conducted in accordance with the National Institutes of Health Guide for the Care and Use of Laboratory Animals and were approved by Northwestern University Animal Care and Use Committee. 


\section{A CA Fields of the Hippocampus Bregma -2.54 $\mathrm{mm}$ (8 weeks)}
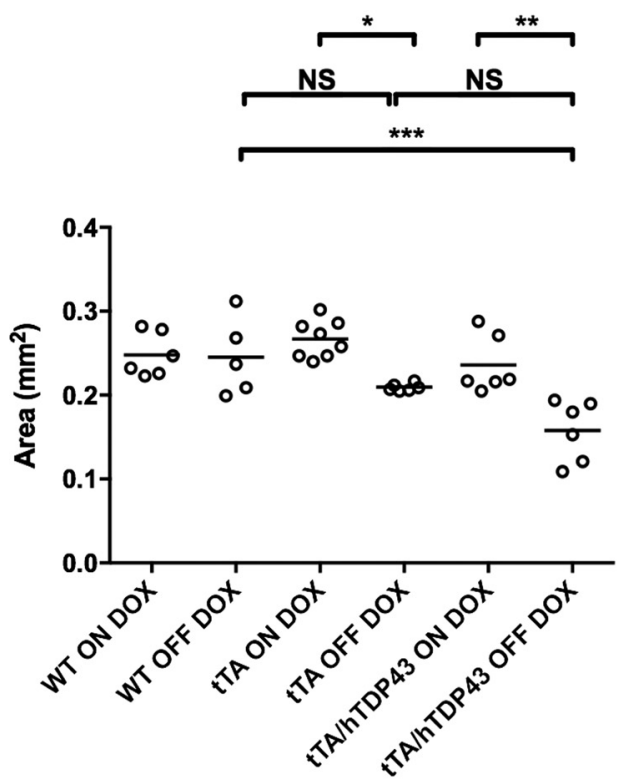

B CA Fields of the Hippocampus
Bregma $-3.40 \mathrm{~mm}$ (8 weeks)

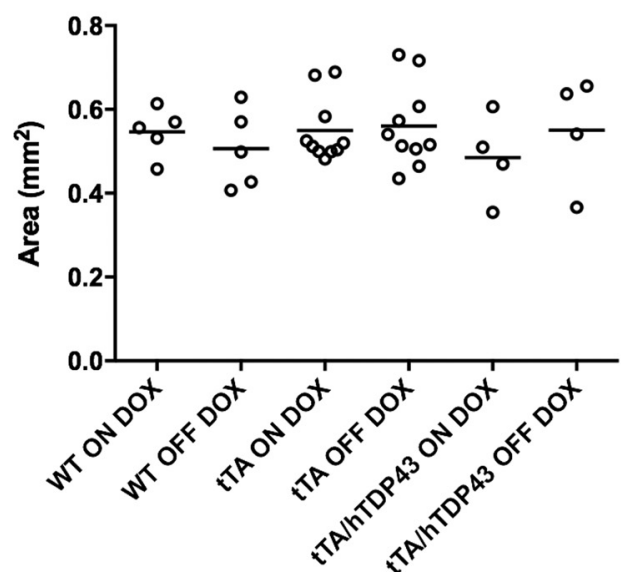

\section{CA Fields of the Hippocampus \\ Bregma -2.54 mm (24 weeks)}
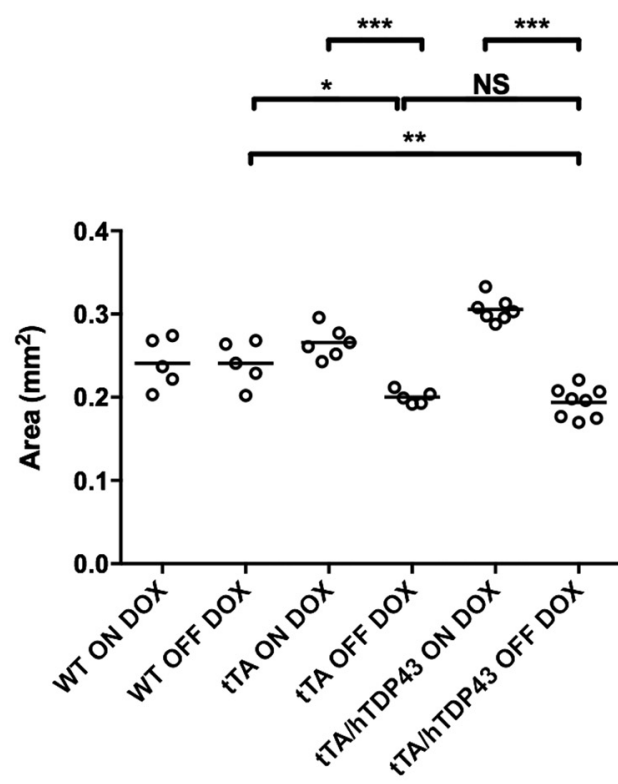

CA Fields of the Hippocampus
Bregma $-3.40 \mathrm{~mm}$ (24 weeks)

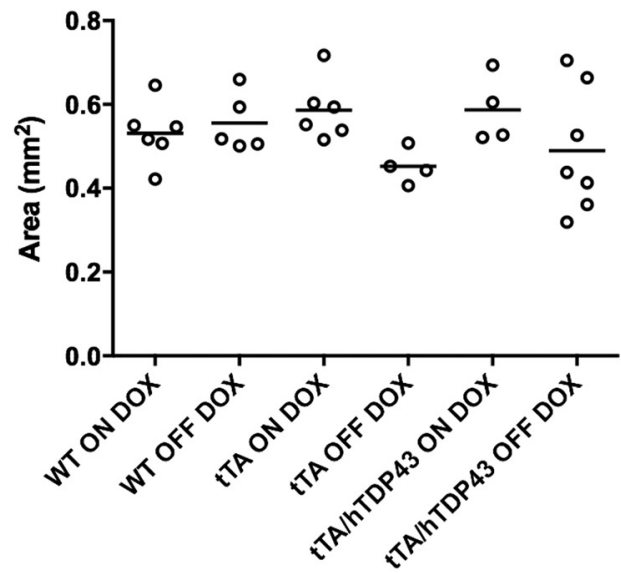

Figure 4. Degeneration in the CA fields of the hippocampus. A, The mid-hippocampal CA fields (represented in bregma $-2.54 \mathrm{~mm}$ ) exhibit significant atrophy in both tTA single transgenic off dox and tTA/hTDP-43 bigenic off dox mice at 8 and 24 weeks postweaning age. The atrophy in the CA fields of the hippocampus in bigenic mice appears similar to tTA single transgenic mice. Treatment with dox in diet eliminates atrophy in the CA fields observed in both tTA single transgenic off dox and tTA/hTDP-43 bigenic off dox mice. $B$, Atrophy is attenuated in the posterior regions (represented in bregma $-3.40 \mathrm{~mm}$ ) of the (A fields at both 8 and 24 weeks postweaning age. $n=4-8$ mice for all genotypes; circles represent individual data points, horizontal lines represent the mean. ${ }^{*} p=0.049-0.019,{ }^{* *} p \leq 0.01,{ }^{* * *} p \leq 0.001$; one-way ANOVA followed by Bonferroni's multiple-comparison test post hoc.

Immunohistochemistry. Frozen coronal whole hemisphere sections of fixed brains were cut at a thickness of $40 \mu \mathrm{m}$ in a 1 in 12 series using a freezing microtome and collected in a 12 -well plate containing $0.1 \mathrm{M}$ phosphate buffer with azide. Series of sections were then stained immunohistochemically using the avidin-biotin-peroxidase method, employing the Vectastain Elite ABC kit (Vectastain Laboratories, Cat- alog \#PK-7100; RRID:AB_2336827). Antibodies to neuronal nuclear protein (NeuN; 1:2000; Milipore, Catalog \#MAB377; RRID: AB_2298772), glial fibrillary acidic protein (GFAP; 1:1500; SigmaAldrich, Catalog \#G9269; RRID:AB_477035), ionized calcium-binding adapter molecule 1 (Iba1; 1:1500; Abcam, Catalog \#ab178846; RRID: AB_2636859), and cleaved caspase-3 (1:500; Abgent, Catalog \#AP3725a; 


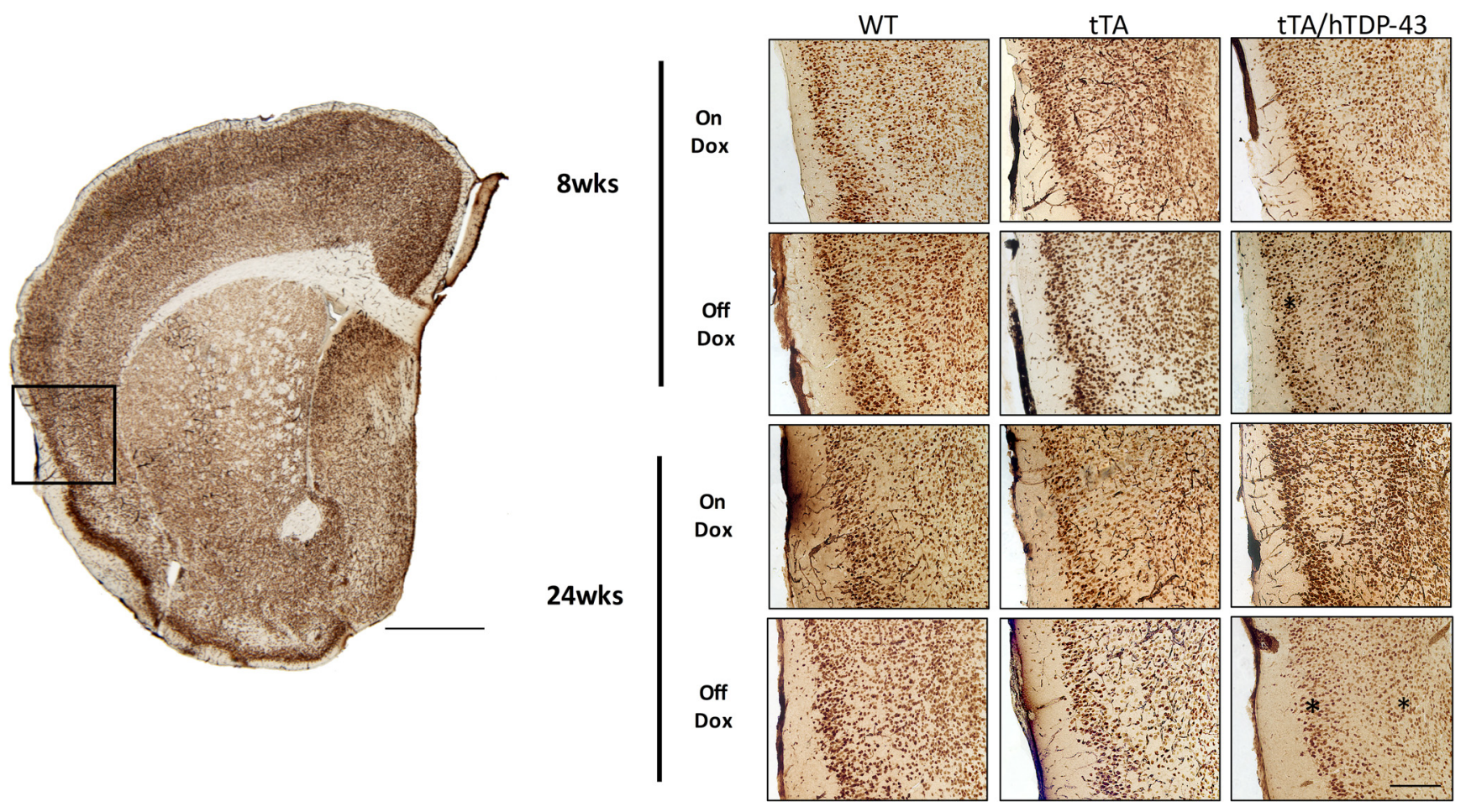

Figure 5. The insula displays atrophy exclusively in TDP-43-expressing bigenic mice. Representative sections stained for NeuN demonstrate degeneration in the insula in tTA/hTDP-43 bigenic mice off dox at 8 or 24 weeks postweaning age. Decrease in neuronal density is clearly visible qualitatively in the insula. Toxicity appears to be hTDP-43-specific because degeneration is rescued in bigenic mice that remain on dox and no atrophy is seen in tTA single transgenic mice off dox diet. Photomicrographs on the right (scale bar, $200 \mu \mathrm{m}$ ) are from the boxed area on the left (scale bar, $500 \mu \mathrm{m})$. *Signify regions of significant neuronal loss.

RRID:AB_10665003) were used. Stained sections were mounted on charged slides, dehydrated in graded alcohols, cleared in xylene, and coverslipped using Sub-X mounting medium.

Cortical area measurements. Micrographs of each mounted section were acquired at $1 \times$ magnification and selected for quantitative analysis based on the presence of anatomical regions of interest. Approximately four sections from each brain were selected based on coordinates from bregma (Paxinos and Franklin, 2001), representing the prefrontal cortex (bregma $2.58 \mathrm{~mm}$ ), cingulate cortex (bregma $0.86 \mathrm{~mm}$ ), motor and somatosensory cortices (bregma $0.86 \mathrm{~mm}$ ), insula (bregma $0.86 \mathrm{~mm}$ ), piriform olfactory cortex (bregma $0.86 \mathrm{~mm} ;-2.54 \mathrm{~mm}$ ), entorhinal cortex (bregma $-2.54 \mathrm{~mm} ;-3.40 \mathrm{~mm}$ ), DG and CA1-3 fields of the hippocampus (bregma $-2.54 \mathrm{~mm} ;-3.40 \mathrm{~mm}$ ). As a control region, which does not show degeneration in FTLD, the cerebellum (bregma $-6.55 \mathrm{~mm}$ ) was also included in this analysis. The boundaries of each cortical region were determined according to the stereotaxic atlas of Paxinos and Franklin (2001; Fig. 1B). Four to five animals were examined for each age and genotype. Area measurements of cortical regions were obtained using ImageJ software to evaluate cortical atrophy. The identities of mouse genotypes were unknown to the person conducting this analysis. Each region was quantified twice and the two measures were averaged to ensure consistency and accuracy of analysis.

Unbiased stereological quantitation. Unbiased stereological quantitation was conducted to determine the total counts of NeuN-immunoreactive neurons, quiescent and activated microglia recognized by Iba1, and astrocytes expressing GFAP in the dentate gyrus. The granule cell layer of the dentate gyrus was quantified. For each genotype, five animals were assessed using the optical fractionator method in the StereoInvestigator software (MBF Bioscience; RRID:SCR_002526). The observer was blind to animal number and genotype throughout the quantitation process. The boundaries of the dentate gyrus was delineated at $4 \times$ magnification, and counting was performed at $60 \times$.

In vivo MRI. All MRI (magnetic resonance imaging) procedures were performed by Northwestern University's Center for Advanced Molecular Imaging. Bigenic mice on or off dox aged for 8 weeks following weaning and anesthetized in an induction chamber with 3\% inhaled isoflurane in oxygen were transferred to a dedicated animal imaging bed with $1 \%$ isoflurane delivered via nosecone. Temperature support was provided via a warm water-circulating blanket, and respiration was monitored using a pneumatic pressure sensor pillow placed under the abdomen (SA Instruments).

Mice were imaged on a 9.4T Bruker BioSpec MRI system (Bruker BioSpin) using a $40 \mathrm{~mm}$ quadrature volume coil. Localizer images were acquired, and then shimming over the brain was performed using a field map-based algorithm (MAPSHIM). 3D images of the brain were acquired using a respiratory gated $3 \mathrm{D}$ gradient echo sequence (FLASH) with TR/TE $/ \alpha=40 \mathrm{~ms} / 10 \mathrm{~ms} / 8^{\circ}$, a $20 \times 16.7 \times 20 \mathrm{~mm}^{3}$ field-of-view, and a matrix size of $166 \times 139 \times 66$ for an isotropic resolution of 120 $\mu \mathrm{m}^{3}$. Three repetitions of this sequence were acquired and averaged to generate adequate signal-to-noise ratio. The nominal scan time was 15 min per repetition, which increased to $\sim 25 \mathrm{~min}$ after allowing for gating efficiency.

Manual MRI segmentation of hippocampus and insular cortex. Scans were opened with the ROI Editor component of the DTI Studio software suite (Jiang et al., 2006). ROI Editor allows for manual segmentation of regions-of-interest (ROIs), and calculates the area of the ROIs in individual slices from MRI. Additionally, it allows for the visualization of ROIs overlaid on the scans in axial, sagittal, and coronal planes, so that contiguity of the scans can be ascertained. The hippocampus and insular cortex, which showed reductions in area in our histological analysis, in accordance to a stereotaxic atlas (Paxinos and Franklin, 2001), were analyzed. The hippocampus traversed twenty $0.12-\mathrm{mm}$-thick coronal slices, and the insular cortex traversed 27 of these slices. Segmentation was performed by an investigator who was blind to the dox treatment status of the experimental animals. For assessment of reliability, segmentation of the insular cortex in four scans was repeated on the day following the initial segmentation, and entire hippocampal volumes were delineated for all 12 scans. Areas of single slices through the midsagittal plane were used as proxies for total brain volume.

Morris water maze. To test hippocampus-dependent memory, the reference memory version of the Morris water maze was used (Morris et al., 
A
Insular Cortex Bregma .86 mm (8 weeks)
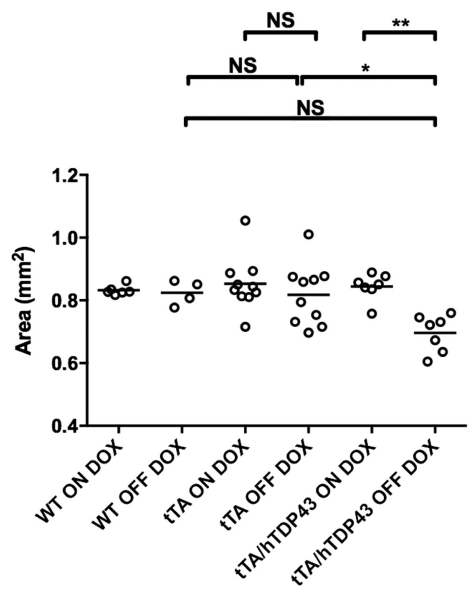

B Bregma .86 mm (8 weeks)
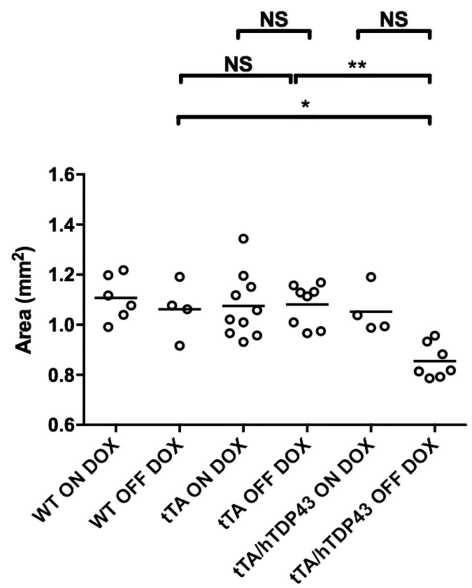

C

Piriform Cortex

Bregma -2.54 mm (8 weeks)
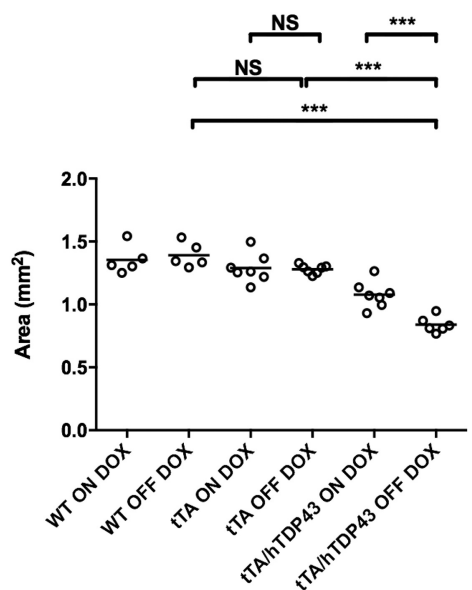

Piriform Cortex
Insular Cortex Bregma $.86 \mathrm{~mm}$ (24 weeks)
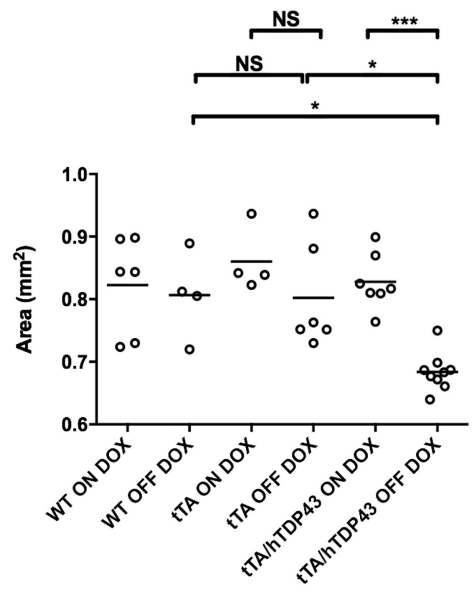

Piriform Cortex Bregma .86 mm (24 weeks)
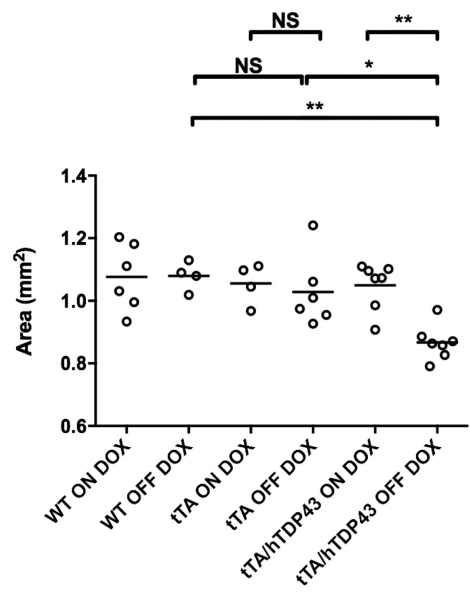

Piriform Cortex Bregma -2.54 mm (24 weeks)
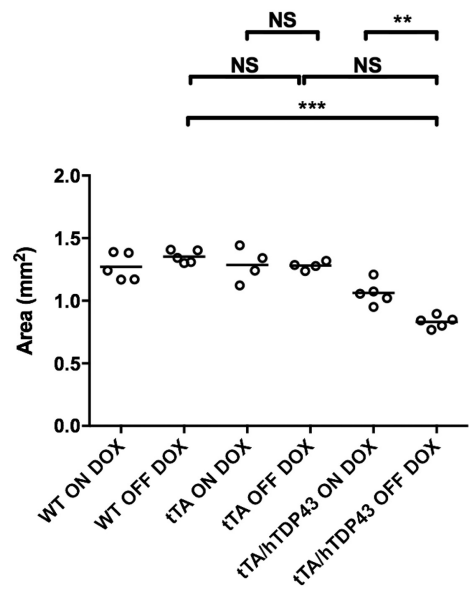

Figure 6. hTDP-43 overexpression causes degeneration in the insula and piriform cortex. $A$, Atrophy is observed in the insula of bigenic mice (tTA/hTDP-43) off dox overexpressing hTDP-43 at 8 or 24 weeks postweaning age. Note that the insula does not show signs of atrophy due to tTA expression alone. $\boldsymbol{B}$, Bigenic mice off dox display significant atrophy in the anterior portion of the piriform cortex (represented in bregma $0.86 \mathrm{~mm}$ ) at 8 or 24 weeks postweaning age with no tTA-dependent toxicity in this cortical region. C, Posterior area of the piriform cortex (represented in bregma $-2.54 \mathrm{~mm}$ ) in bigenic mice also shows significant atrophy at 8 or 24 weeks postweaning age. tTA expression alone causes no significant atrophy. $n=4-8$ mice for all genotypes; circles represent individual data points, horizontal lines represent the mean. ${ }^{*} p=0.034-0.011,{ }^{* *} p \leq 0.01,{ }^{* * *} p \leq 0.001$; one-way ANOVA followed by Bonferroni's multiple-comparison test post hoc.
1982). Animals were aged 8 or 24 weeks postweaning on or off dox diet and tested four times per day over a $6 \mathrm{~d}$ period with intertrial intervals of $1 \mathrm{~h}$ (Lindner et al., 1992). For this test, the first $3 \mathrm{~d}$ were used as training days. Initially, a platform protruded out of the surface of the water. Once the mouse was able to locate the platform, training continued but the platform was submerged. The final $3 \mathrm{~d}$ were used as test days. During this time the water in the maze was opaque and the platform was submerged. The platform remained in the same location throughout testing, but the starting position varied for each trial. Time spent locating the platform was used as a measure of reference memory. In each testing trial, the maximum swim time allowed was $120 \mathrm{~s}$.

Statistical analyses. Numbers of animals per group are indicated in figure legends. GraphPad Prism (RRID:SCR_002798) was used for most statistical analysis except for MRI imaging volume results, for which the SPSS software was used (IBM; RRID:SCR_002865). The statistical significance between means of experimental and control groups was determined using the two-tailed Student's $t$ test. For comparisons involving more than two groups, one-way or two-way ANOVA were used as appropriate, followed by Bonferroni's multiplecomparison post hoc tests. Data are presented as measures for each animal and group means, and $p<0.05$ was considered significant. Stereological data were similarly analyzed using one-way ANOVA followed by Bonferroni's multiple-comparison post hoc tests.

\section{Results}

We report on a biallelic mouse model that uses tTA to drive the expression of human TDP-43 allele. This model system results in overexpression of hTDP-43 and formation of round intracytoplasmic inclusions (Cannon et al., 2012). However, the effect of tTA expression in this mouse model had not been investigated. We obtained brains from monogenic and bigenic offspring of these two alleles to determine independent toxicity due to expression of tTA and hTDP-43 (Fig. 1A). Animals were aged to 8 and 24 weeks postweaning while remaining on or off dox diet. We investigated atrophy as an indicator of neurodegeneration in the hippocampus, cortical limbic areas, and several neocortical regions in these animals (Fig. 1B).

\section{Neurodegeneration due to tTA}

tTA toxicity in hippocampal subregions In accordance with past studies (Han et al., 2012), hippocampal degeneration was observed in single tTA transgenic and tTA/hTDP-43 bigenic mice. A tTA-dependent toxicity emerged where tTA expression led to degeneration of the hippocampus, in particular thinning of the molecular layer of the DG and the py- 
ramidal cell layer of the CA fields. Interestingly, the degeneration was rescuable with dox in the mouse diet (Fig. 2). Using NeuN-stained coronal sections, we analyzed the area of the DG in sections at the mid-hippocampal formation harvested at 8 and 24 weeks postweaning age. We quantified the area of the DG in bigenic, tTA single transgenic and WT mice on or off dox diet (Fig. 3). The area of the DG in tTA mice off dox was $44 \%$ smaller than in age-matched WT mice at 8 weeks postweaning age $(p<0.001)$. Furthermore, tTA-expressing mice demonstrated a $30 \%$ reduction in area of the DG compared with tTA mice that received dox throughout the experiment at 8 weeks postweaning age $(p=0.027)$. Degeneration of DG associated with tTA toxicity was more concentrated in the midhippocampal region and, to a lesser extent, in the posterior region of the DG in tTA mice off dox compared with agematched WT mice at 8 weeks postweaning age $(p>0.05)$. Similar patterns of atrophy were obtained at 24 weeks postweaning age in the mid-hippocampal $(p<$ $0.001)$ and posterior hippocampus $(p>$ $0.05)$. However greater levels of atrophy were observed at later ages.

We also found significant degeneration in CA1-3 of the hippocampus in tTA single transgenic mice (Fig. 4). Similar to the DG, toxicity in the CA fields was concentrated in the mid-hippocampal region and was present at $8(p>0.05)$ and 24 weeks $(p>0.05)$ postweaning age compared with WT control mice on or off dox diet. No toxicity was observed in the CA fields in tTA-expressing mice off dox when a more posterior region of the hippocampus was examined $(p>0.05)$.

tTA toxicity in other limbic structures Given the toxicity due to expression of tTA in the hippocampus, a core limbic structure, we also examined potential toxicity of tTA in other limbic cortical areas (Fig. 5). The insula $(p>0.05)$, entorhinal cortex $(p>0.05)$, cingulate cortex $(p>$ $0.05)$, and piriform olfactory cortex $(p>$ $0.05)$ were examined at 8 and 24 weeks postweaning age in animals on or off dox diet (Fig. 6). No atrophy was observed in any of these cortical areas in tTA single transgenic mice compared with wild-type control mice. (Fig. 7).

tTA toxicity in neocortical regions

Next we examined potential degeneration due to tTA in neocortical regions. The dorsolateral prefrontal cortex, motor cortex and somatosensory cortex were examined in mice at 8 or 24 weeks postweaning
A

\section{Entorhinal Cortex Bregma -2.54 mm (8 Weeks)}

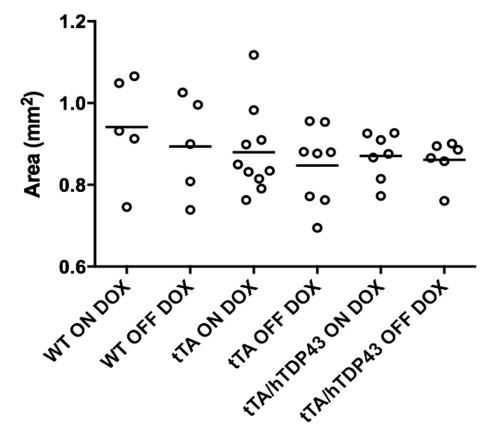

B
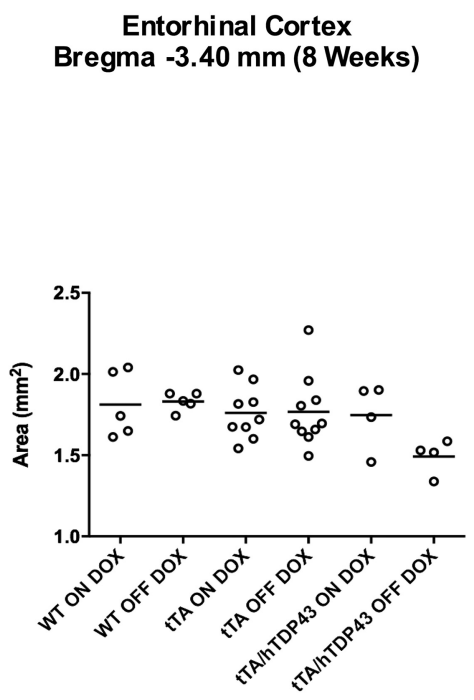

C

$$
\begin{gathered}
\text { Cingulate Cortex } \\
\text { Bregma } .86 \mathrm{~mm} 8 \text { weeks }
\end{gathered}
$$

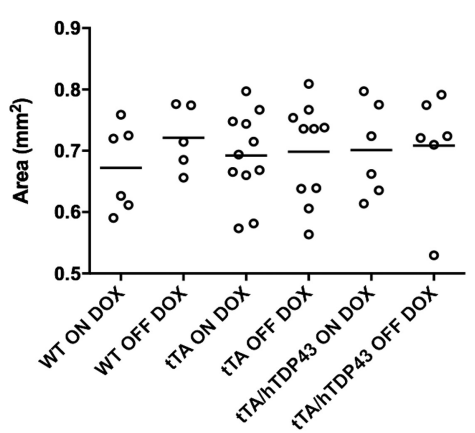

Entorhinal Cortex

Bregma -2.54 mm (24 Weeks)

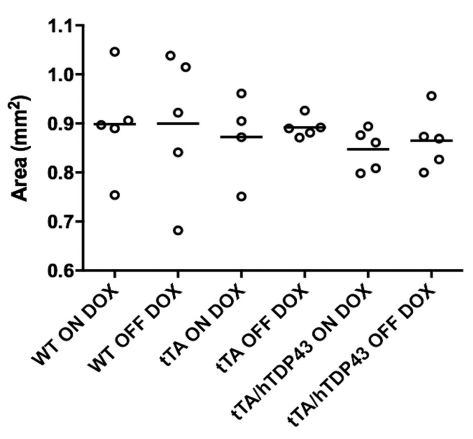

Entorhinal Cortex Bregma $-3.40 \mathrm{~mm}$ (24 Weeks)
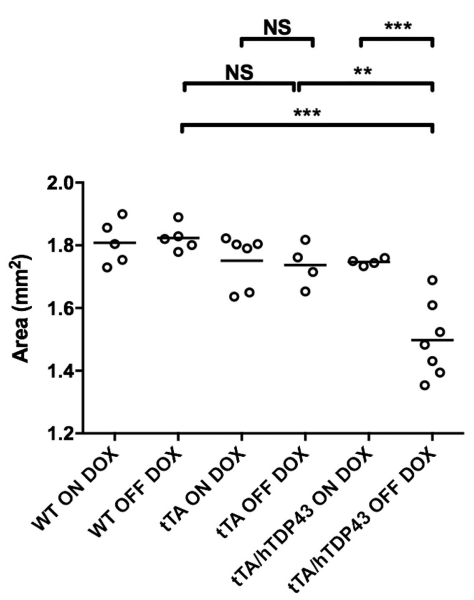

Cingulate Cortex Bregma $.86 \mathrm{~mm} 24$ weeks
Figure 7. hTDP-43 overexpression leads to degeneration in the posterior entorhinal cortex but not the cingulate cortex. $A$, Area measurement analysis of the anterior region of the entorhinal cortex (represented in bregma $-2.54 \mathrm{~mm}$ ) shows no atrophy due to hTDP-43 or tTA overexpression at either 8 or 24 weeks postweaning age. $\boldsymbol{B}$, The posterior region of the entorhinal cortex (represented in bregma $-3.40 \mathrm{~mm}$ ) displays significant cortical atrophy due to hTDP-43 but not tTA overexpression in 24 weeks postweaning age bigenic mice. C, The cingulate cortex (represented in bregma $0.86 \mathrm{~mm}$ ) is unaffected by hTDP-43 or tTA overexpression at both 8 and 24 weeks postweaning ages. $n=4-8$ mice for all genotypes; circles represent individual data points, horizontal lines represent the mean; ${ }^{* *} p \leq 0.01,{ }^{* * *} p \leq 0.001$, one-way ANOVA followed by Bonferroni's multiple-comparison test post. 


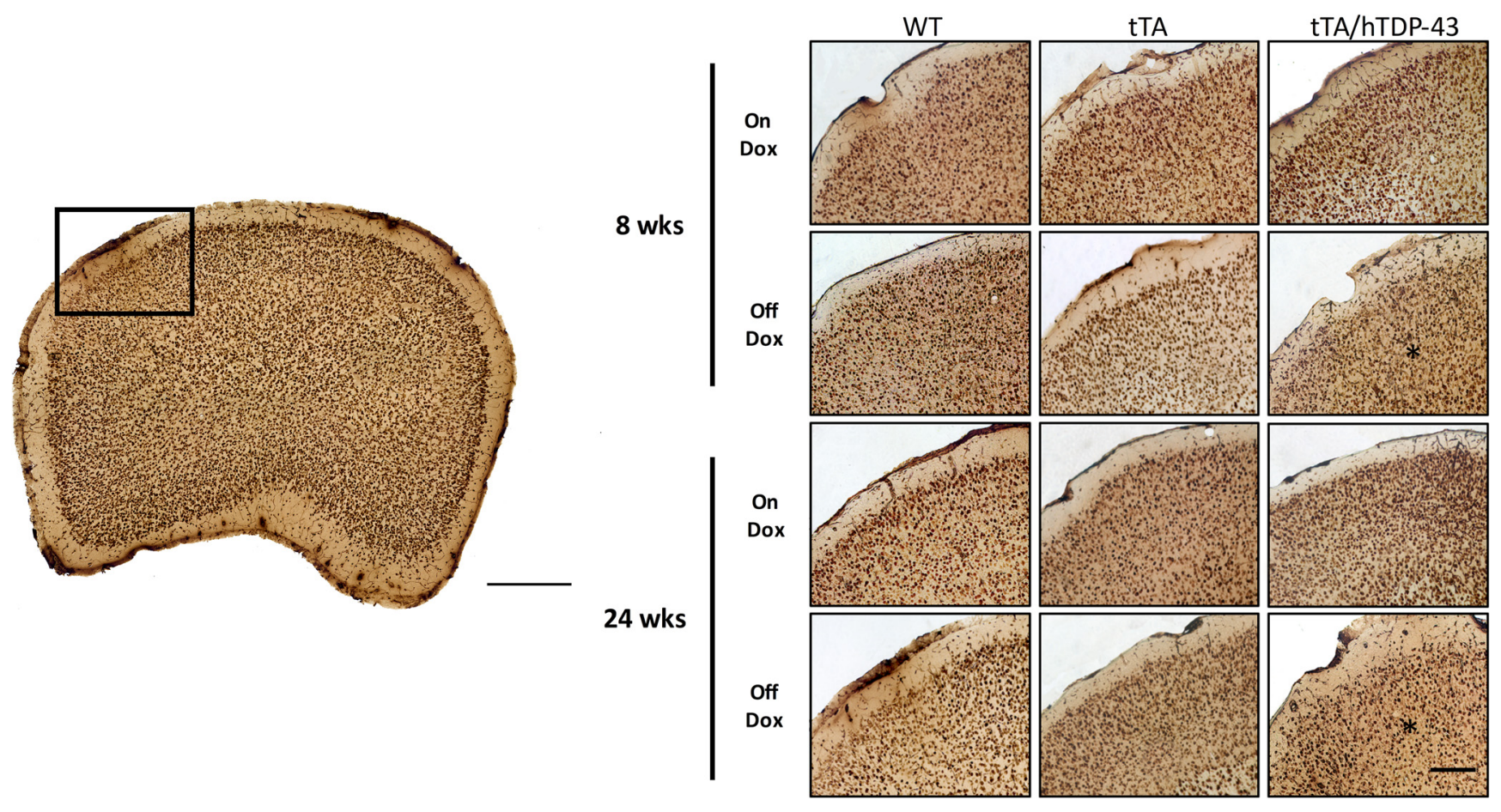

Figure 8. Degeneration of the dorsolateral prefrontal cortex in bigenic mice. Representative sections stained for NeuN in bigenic animals (tTA/hTDP-43) off dox demonstrate significant atrophy of the dorsolateral prefrontal cortex. Atrophy is apparent at 8 weeks postweaning age, and worsens at 24 weeks. No atrophy is apparent in the tTA single transgenic mice, or control bigenic mice, that remain on doxycycline diet. Magnified photomicrographs on the right (scale bar, $200 \mu \mathrm{m}$ ) are from the region represented in inset of photomicrograph on the left (scale bar, $500 \mu \mathrm{m})$. Asterisk ${ }^{*}$ ) represents region of visible neuronal loss.

age on or off dox (Fig. 8). No significant atrophy was observed in tTA single transgenic mice compared with wild-type animals (Fig. 9; $p>0.05$ for all comparisons).

\section{Degeneration due to hTDP-43}

Next, we explored the possibility that tTA-dependent toxicity in our mice is selective to the hippocampus, whereas hTDP-43dependent toxicity is selective to cortical regions (Cannon et al., 2012). We predicted this outcome based on the fact that atrophy of the hippocampus is not a characteristic feature of FTLD (Josephs, 2008). Hippocampal neuronal damage is usually linked to episodic memory deficits that are seen in Alzheimer's disease. FTLD commonly causes apathy, loss of emotional control, and language dysfunction, which are attributable to degeneration of frontal and temporal cortices, including limbic structures other than the hippocampus (Brettschneider et al., 2014).

Degeneration in hippocampal subregions of bigenic mice tTA/hTDP-43 bigenic mice off dox displayed significant degeneration in the DG compared with WT mice as well as tTAexpressing mice on dox diet. (Fig. 3). There appeared to be specific loss of granule cells within the granular layer of the DG at both 8 and 24 weeks postweaning age (Fig. 2). Atrophy observed at 8 weeks continued at 24 weeks postweaning age with significant reduction of 55\% in the area of DG in bigenic mice off dox compared with age-matched WT mice $\left(8\right.$ weeks: $p<0.001, F_{(5,36)}=$ $11.41 ; 24$ weeks: $\left.p<0.001, F_{(5,30)}=19.05\right)$. The DG degeneration affected more of the mid-region (bregma $-2.54 \mathrm{~mm}$ ) than posterior region (bregma $-3.40 \mathrm{~mm}$ ) of the hippocampus (Fig. 3). Interestingly, there was small but significant atrophy in DG of posterior hippocampal regions in bigenic animals off dox at 24 weeks postweaning age compared with WT animals, indicating that TDP-43 expression may result in additional toxic effects in this region $\left(p=0.022, F_{(5,27)}=48.13\right.$; Fig. 3$)$. Furthermore, the examination of the CA fields revealed results comparable to those obtained for DG. Both bigenic and tTA mice off dox showed a statistically significant loss of CA1-3 area compared with WT mice at 8 and 24 weeks of expression ( 8 weeks: $p<0.001, F_{(5,31)}=$ $10.61 ; 24$ weeks: $p<0.01, F_{(5,31)}=28.94$; Fig. 4). Overall, atrophy seen in the hippocampus in bigenic and tTA single transgenic mice was rescuable when animals were fed dox diet (Figs. 3, 4).

\section{Degeneration in other limbic regions of bigenic mice}

A common clinical feature of FTD is dysregulation of emotional behavior (Balconi et al., 2015). Because the limbic system is chiefly involved in emotional control and regulation, we also examined several limbic structures in tTA/hTDP-43 bigenic animals and tTA single transgenic mice.

Examination of insular cortex revealed significant degeneration in bigenic mice off dox at 8 and 24 weeks postweaning age compared with animals from the rest of the cohort (Fig. 6). This degeneration was statistically significant compared with tTA mice at 8 weeks as well as tTA and WT mice after 24 weeks postweaning ages (tTA mice at 8 after 8 weeks: $p=0.0181, F_{(5,38)}=$ 4.86; tTA mice after 24 weeks: $p=0.0114, F_{(5,30)}=7.75$; WT mice after 24 weeks: $\left.p=0.0284, F_{(5,30)}=7.75\right)$. We also examined the anterior and posterior regions of the piriform olfactory cortex in bigenic animals at 8 and 24 weeks postweaning ages ( 8 weeks: $p=$ $0.0247, F_{(5,33)}=5.915 ; 24$ weeks: $\left.p<0.01, F_{(5,28)}=6.029\right)$. Similar to the insula, we observed significant atrophy in both regions of piriform cortex in bigenic mice off dox compared with the group of animals from different conditions (Fig. 6). Interestingly, the more posterior regions of the piriform cortex showed more significant atrophy at 8 and 24 weeks unlike the pattern of atrophy in the hippocampus ( 8 weeks: $p<0.001, F_{(5,31)}=30.38 ; 24$ weeks: $p<$ $\left.0.001, F_{(5,22)}=25.6\right)$. 
A

Frontal Cortex Bregma $2.58 \mathrm{~mm}$ (8 weeks)
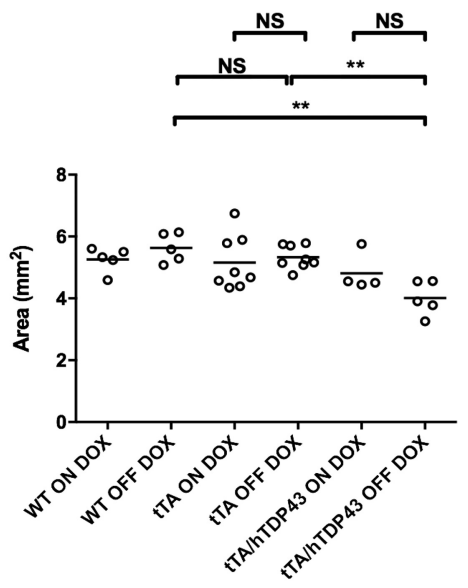

B

Somatosensory Cortex Bregma .86 mm (8 weeks)

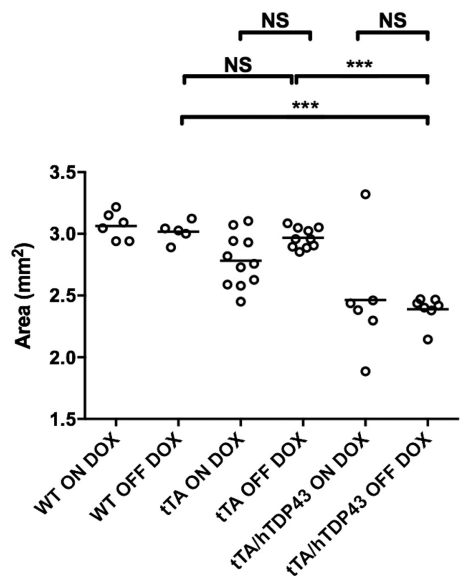

C
Bregma $.86 \mathrm{~mm}$ (8 weeks)

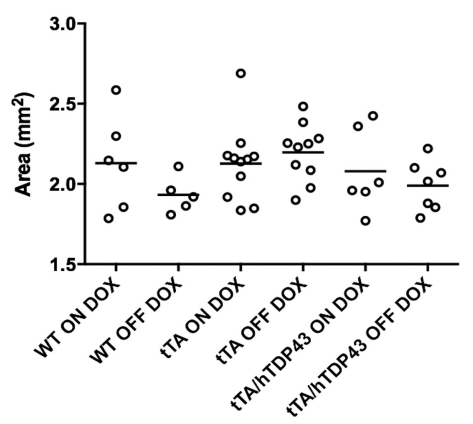

Frontal Cortex Bregma $2.58 \mathrm{~mm}$ (24 weeks)

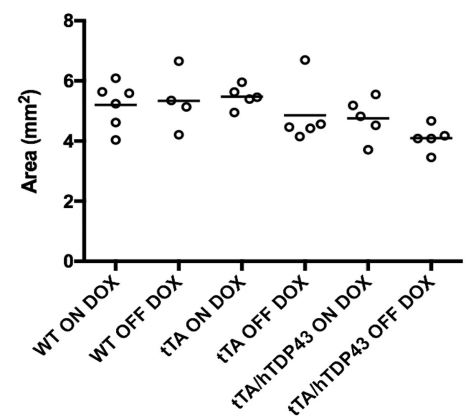

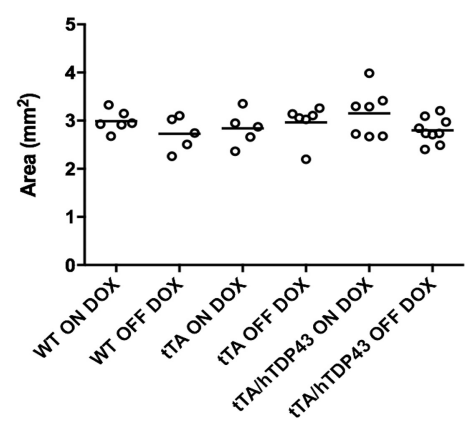

\section{Motor Cortex Bregma $.86 \mathrm{~mm}$ (24 weeks)}

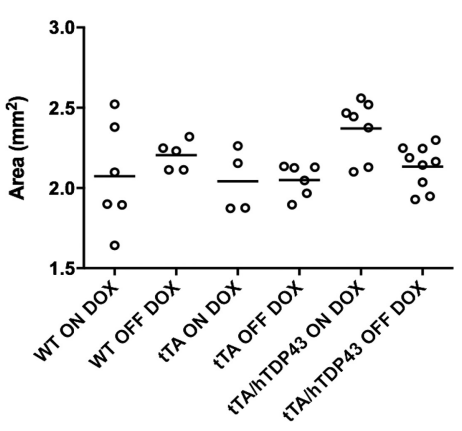

Analysis of the entorhinal cortex revealed significant degeneration due to hTDP-43 overexpression in posterior but not anterior aspects of this region. The loss of area appeared to be only a feature of 24 weeks postweaning age bigenic mice $\left(p<0.001, F_{(2,25)}=13.72\right)$. After 8 weeks of expression, neither anterior nor posterior regions of the entorhinal cortex revealed significant atrophy (anterior: $p>$ $0.05, F_{(5,35)}=0.6461$; posterior: $p>0.05$, $\left.F_{(5,31)}=2.023\right)$. Finally, the cingulate cortex showed no significant degeneration at 8 or 24 weeks postweaning age in bigenic mice compared with the other groups of animals ( 8 weeks: $p>0.05, F_{(5,38)}=$ 0.2706 ; 24 weeks: $p>0.05, F_{(5,30)}=$ 0.9751; Fig. 7)

\section{Degeneration due to hTDP-43 in} neocortical regions

In FTLD, the prefrontal cortex displays significant atrophy. Accordingly, we examined the dorsolateral prefrontal cortex in bigenic mice off dox. Bigenic animals displayed significant atrophy in this region compared with WT or tTA mice off dox, and bigenic mice on dox after 8 weeks of transgene expression $(p<0.01$, $F_{(5,29)}=4.631$; Fig. 9). At 24 weeks postweaning age, bigenic mice showed only a nonsignificant trend toward atrophy in this region compared with the other groups $\left(p>0.05, F_{(5,24)}=2.24\right)$.

Two final cortical areas examined in bigenic mice were the somatosensory and motor cortices (Fig. 9). The somatosensory cortex displayed significant atrophy in bigenic mice off dox after 8 weeks of TDP-43 overexpression compared with tTA off dox $\left(p<0.001, F_{(5,39)}=12.21\right)$. However, this difference was no longer present in mice at 24 weeks postweaning age $\left(p>0.05, F_{(6,32)}=1.217\right)$. The motor cortex showed no significant atrophy in any of the groups of animals at 8 or 24 weeks of expression ( 8 weeks: $p>0.05$, $F_{(5,39)}=1.449 ; 24$ weeks: $p>0.05, F_{(5,31)}=$ 2.851)

To examine the specificity of the results obtained in cortical regions, the cerebellum, an area not affected in FTLD was also examined. The area of the cerebellum displayed no significant differences among the three groups of animals at 8 or 24 weeks
Figure 9. Bigenic mice display degeneration in the dorsolateral prefrontal cortex and somatosensory cortex but not in the motor cortex. A, Bigenic mice (tTA/hTDP-43) off dox display significant atrophy at 8 weeks of hTDP-43 transgene expression in the dorsolateral prefrontal cortex. Area measurement shows that atrophy is due to hTDP-43 expression as no atrophy is seen in control bigenic mice that remain on dox diet or the tTA single transgenic mice. By 24 weeks postweaning age, hTDP-43 toxicity in the dorsolateral prefrontal cortex attenuates and is no longer significantly different from the rest of the cohort of mice. $\boldsymbol{B}$, The somatosensory cortex shows a similar pattern of atrophy to dorsolateral prefrontal cortex. hTDP-43 toxicity is present at 8 weeks postweaning age, but is no longer detectable in 24 weeks postweaning age bigenic mice. Additionally, in contrast to other regions, the bigenic mice at 8 weeks do not show recovery in somatosensory cortex by remaining on the dox diet. C, The motor cortex shows

\section{$\leftarrow$}

no degeneration due to hTDP-43 or tTA at 8 or 24 weeks postweaning age. $n=4-8$ mice for all genotypes; circles represent individual data points, horizontal lines represent the mean; ${ }^{* *} p \leq 0.01,{ }^{* * *} p \leq 0.001$, one-way ANOVA followed by Bonferroni's multiple-comparison test post hoc. 
A
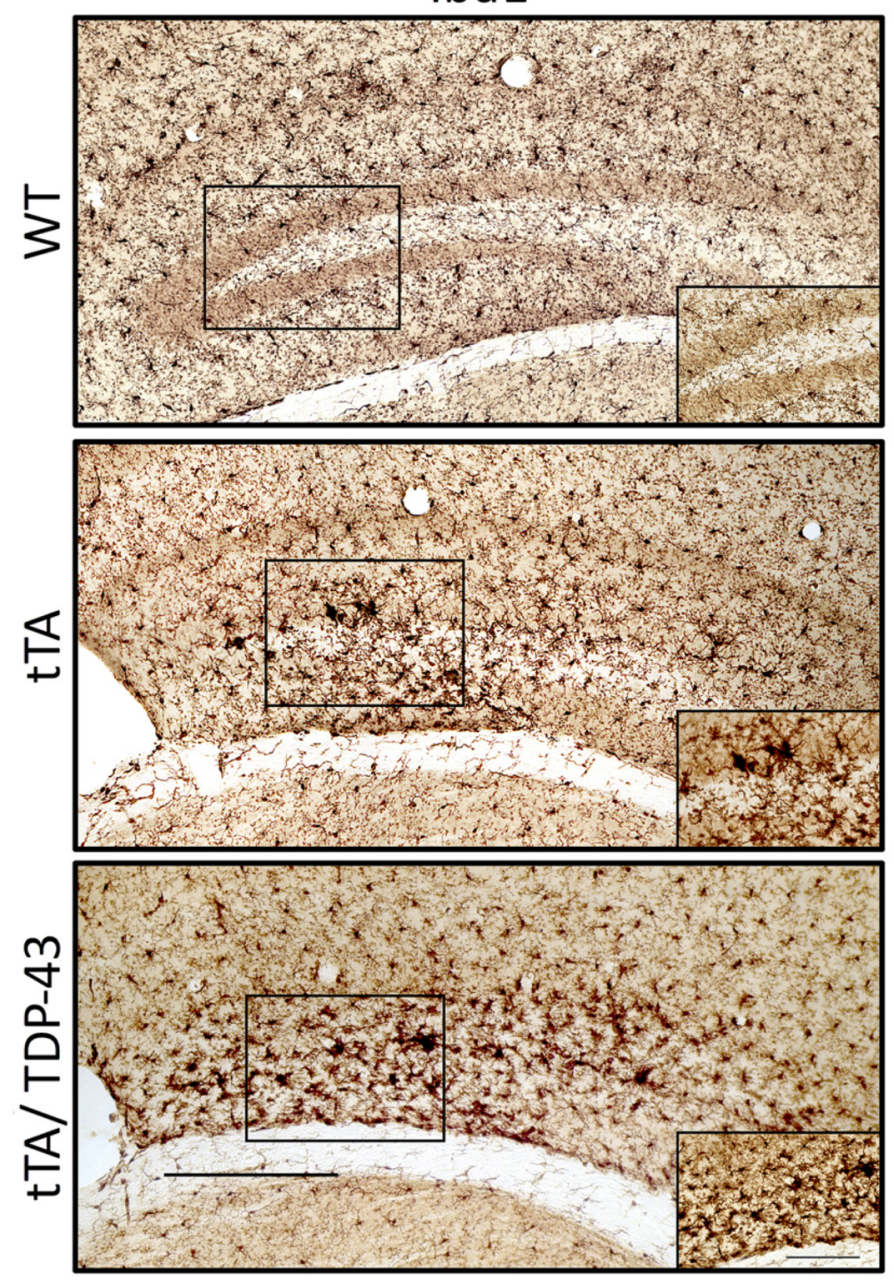

B

GFAP- Postive Astrocytes

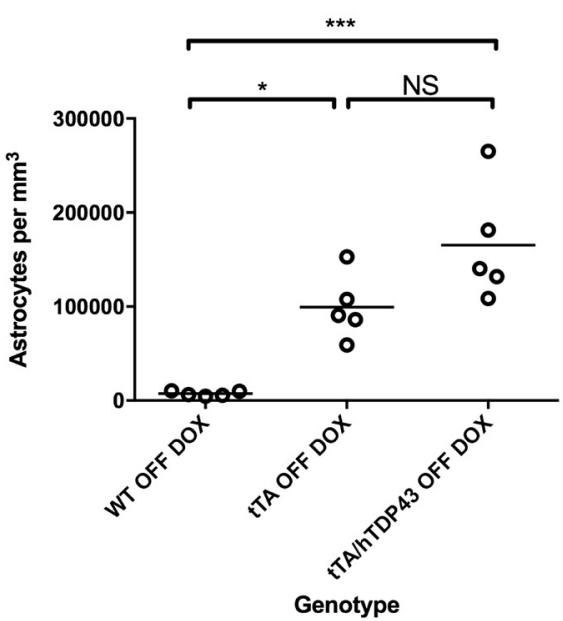

GFAP
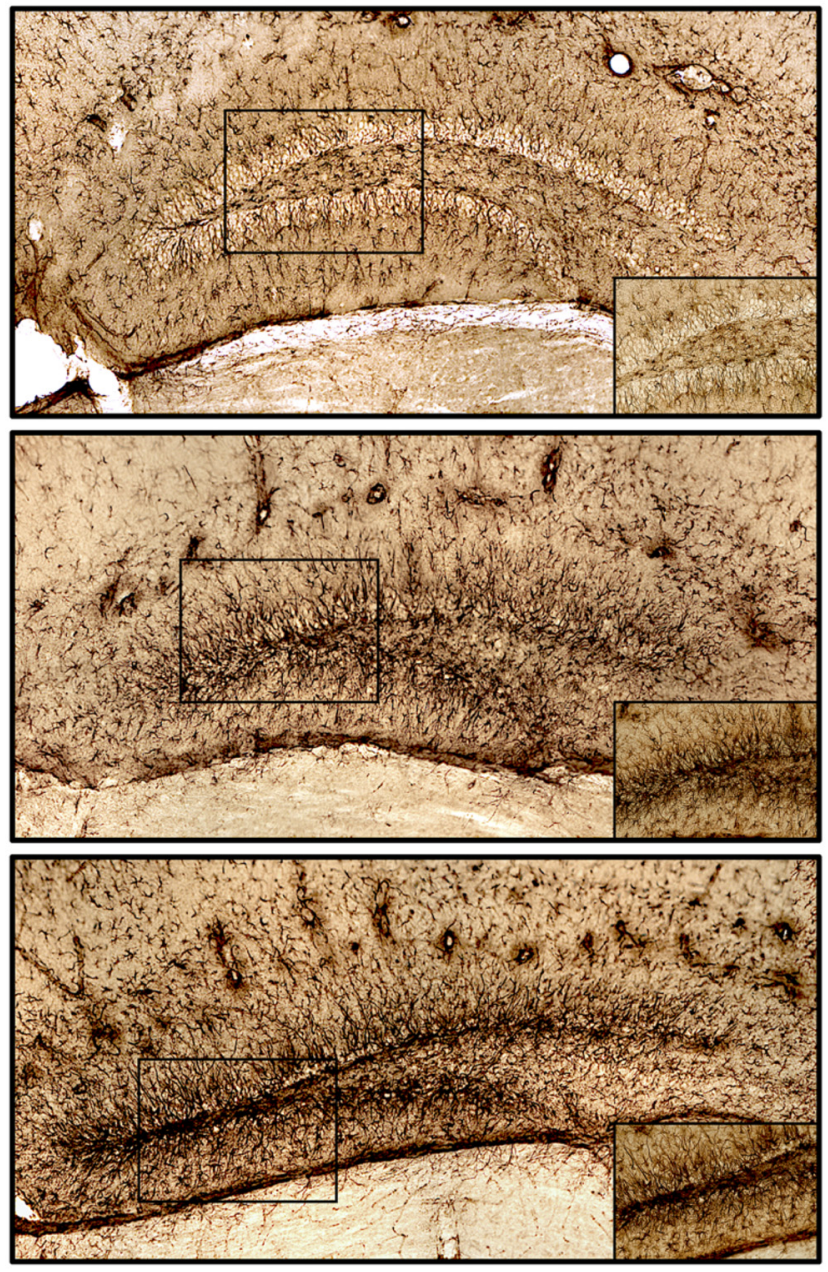

C

Iba1- Postive Microglia

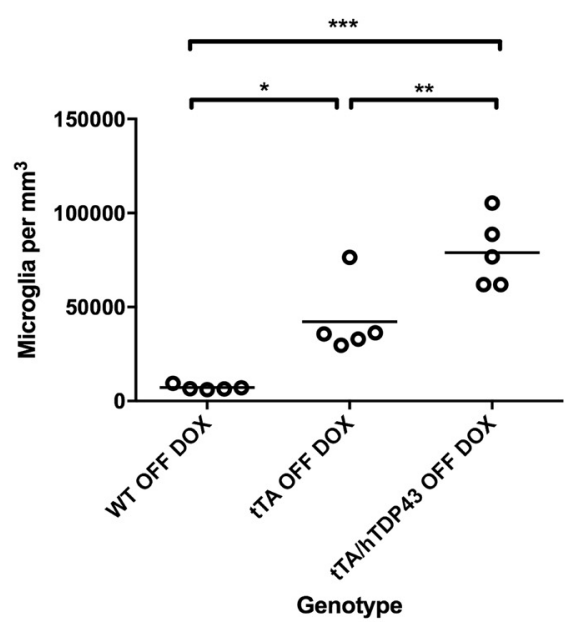

Figure 10. tTA expression causes inflammatory responses in the hippocampus. A, Immunostaining using antibodies specific to GFAP, a marker of astrocytes, and Iba1, a marker of microglia, on coronal brain sections $(40 \mu \mathrm{m})$ of 24 weeks postweaning age mice show increased positivity when tTA is expressed in bigenic (tTA/hTDP-43) mice off dox and tTA single transgenic mice off dox. The molecular layer of the dentate gyrus where granule cells reside displays significant accumulation of hypertrophied microglia and astrocytes. WT control mice show minimal immunoreactivity to GFAP and Iba1. Scale bar, $30 \mu \mathrm{m}$. Insets, The boxed area on the left. Scale bar, $20 \mu \mathrm{m}$. B, C, Unbiased stereological quantitation determined the density of microglia and astrocytes in the granular layer of the dentate gyrus. $\boldsymbol{B}$, Quantitative analyses show a significant increase of GFAP-positive astrocytes in the tTA single transgenic and bigenic compared with WT mice. However, there is no significant difference observed between the bigenic and tTA single transgenic mice. $C$, Analysis of lba1 reactive microglia shows a significant increase in the bigenic and tTA(Figure legend continues.) 


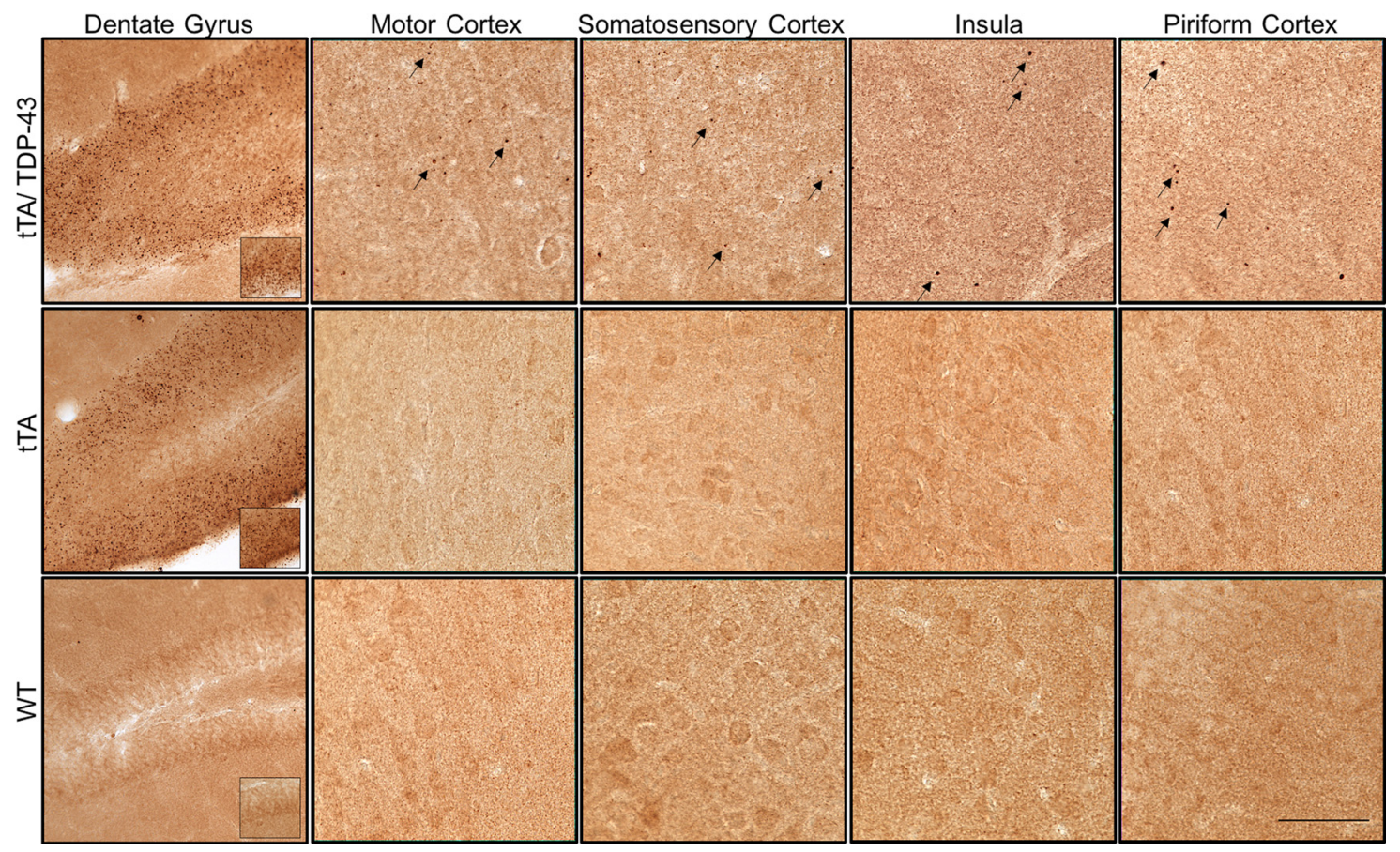

Figure 11. tTA expression causes apoptosis, evident by increased cleaved caspase-3, in the hippocampus, while TDP-43 expression in bigenic mice causes apoptosis beyond hippocampus in various cortical regions. Immunostaining using cleaved caspase-3 neoepitope antibody on coronal brain sections of 24 weeks postweaning age mice demonstrate cleaved caspase-3-positive puncta in the DG and cortical neurons of motor cortex, somatosensory cortex, insula and piriform of bigenic (tTA/hTDP-43) animals off dox (black arrows). Scale bar, $50 \mu \mathrm{m}$. Immunoreactivity for cleaved caspase-3 in tTA single transgenic mice off dox is limited to the dentate gyrus. No immunoreactivity for cleaved caspase-3 is observed in wild-type mice.

postweaning age on dox or off dox $\left(p>0.05 F_{(5,24)}=0.2255\right.$; data not shown).

\section{Inflammation, apoptosis, and loss of neurons}

To further characterize the basis of cortical atrophy, we examined neuroinflammation in bigenics and tTA single transgenic mice. Microglia and astrocytes, which are known key regulators of neuroinflammation, adopt an activated state in response to stress or injury. Microglia become primed and hypertrophied by inflammation (Perry and Holmes, 2014), which can be detected by Iba1 immunopositivity. Astrocytes respond to insults through a process referred to as reactive astrogliosis that can be detected by GFAP immunopositivity (Colombo and Farina, 2016). We focused more specifically on the DG of the hippocampus where atrophy was pronounced in our animal model. After immunostaining for Ibal and GFAP greater densities of hypertrophied microglia and astrocytes were present in the DG of tTA and bigenic mice compared with WT mice at 24 weeks postweaning age (Fig. 10).

We also immunostained coronal brain sections with the cleaved caspase- 3 neoepitope antibody to study caspase- 3 activation, a crucial event in apoptotic cell death, that is a feature of many chronic neurodegenerative diseases (D'amelio et al., 2012). We observed cleaved caspase-3-positive puncta in the closely packed granule cell layer of the DG in both bigenic and tTA single transgenic mice at 24 weeks postweaning age. Interestingly, cleaved caspase-3-positive puncta were also found in cortical re-

\section{$\leftarrow$}

(Figure legend continued.) single transgenic mice compared with WT mice. In addition, a significant increase of microglia is observed in the bigenic mice compared with the tTA single transgenic mice. $n=4-8$ animals per genotype; circles represent individual data points, horizontal lines represent the mean. ${ }^{*} p=0.019-0.011,{ }^{* *} p \leq 0.01$, ${ }^{* * *} p \leq 0.001$; one-way ANOVA test followed by Bonferroni's multiple-comparison test post hoc gions, e.g., motor cortex, somatosensory cortex, insular and piriform cortex, but only in bigenic and not in tTA single transgenic mice and WT controls (Fig. 11).

Next, using stereological counting methods, we quantified granule cells of the DG, the region of the hippocampus that displayed significant atrophy in $\mathrm{tTA}$ single transgenic and bigenic mice. At 24 weeks postweaning age, using NeuN-immunostained sections, both bigenic and tTA-expressing mice off dox showed significant loss of granule cells compared with WT mice off dox $\left(p<0.01, F_{(2,12)}=\right.$ $8.081)$. However, there was no significant difference in the neuron counts between the bigenic and tTA single transgenic mice $(p>$ 0.05 ; Fig. 12). Stereological quantitation of astrocytes revealed similar results. After 24 weeks of expression, the bigenic and tTA single transgenic mice showed significant increase in astrocytes in the dentate gyrus compared with the WT mice $\left(p<0.001, F_{(2,12)}=18.93\right)$. However, the bigenic mice displayed no significant difference in the number of astrocytes compared with the tTA single transgenic mice ( $p>0.05$; Fig. 10B). The number of microglia in the dentate gyrus was significantly higher in the bigenic and tTA single transgenic mice compared with the WT mice $\left(p<0.001, F_{(2,12)}=26.87\right)$. Moreover, the bigenic mice in particular demonstrated a significant increase in the number of microglia compared to the tTA single transgenic mice (Fig. 10C).

\section{tTA induced atrophy in the hippocampus is rescuable in C57BL/6 mouse background}

Previous reports have shown that the tTA transgene effect on hippocampal degeneration can be rescued on C57BL/6 background mice (Han et al., 2012). Therefore, we repeated a similar study to examine atrophy in the hippocampus of WT and tTA single transgenic mice on C57BL/6 background (Fig. 13). These mice expressing tTA were obtained from The Jackson Laboratory on an already established B6 background. We then aged them out 


\section{Total Number (Dentate Gyrus)}

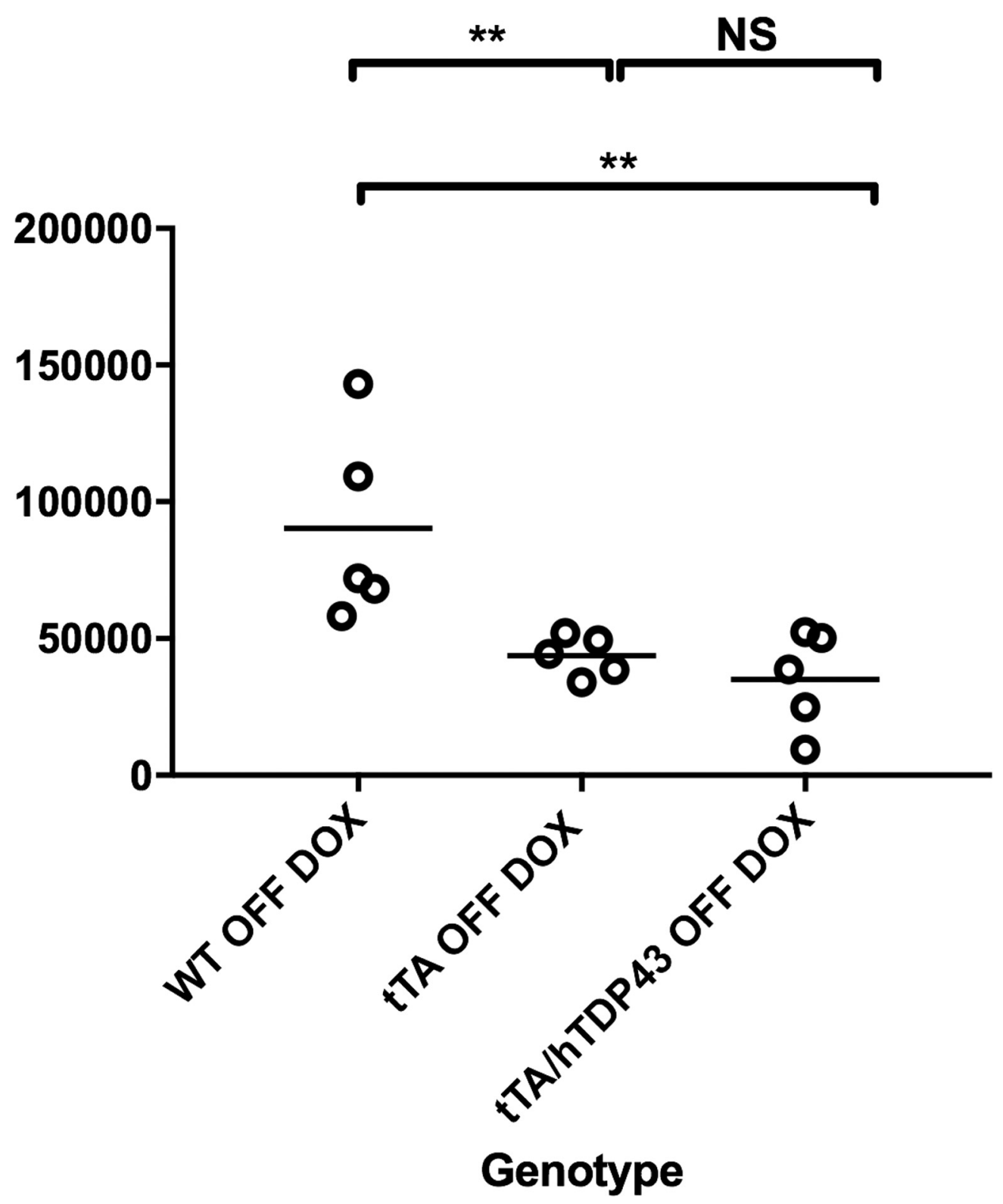

Figure 12. Atrophy in the DG is associated with neuronal loss. Unbiased stereological quantitation was used to determine total count of NeuN-immunoreactive neurons within the granular layer of the DG. Total count of NeuN-positive neurons displayed a significant decrease in 24 week postweaning age bigenic (TTA/TDP-43) off dox mice and the single tTA transgenic off dox mice relative to the WT control mice. The total count of NeuN-positive neurons between bigenic (tTA/TDP-43) off dox mice and the single tTA transgenic off dox mice was not significant (per genotype, $n=4-8)$. ${ }^{* *} p<.01$.

to 8 or 24 weeks postweaning. At 8 weeks postweaning age, DG in tTA/C57BL/6 (tTA/B6)-expressing mice showed no significant atrophy compared with age-matched WT mice. A similar result was obtained in mice 24 weeks postweaning age (Fig. 13). Furthermore, tTA/ B6 mice off dox showed no decrease in DG area compared with age-matched tTA single transgenic mice on dox (tTA/B6, $0.112 \mathrm{~mm}^{2}$; tTA mice on dox, $0.109 \mathrm{~mm}^{2}$ ).

We also examined the CA fields in tTA/B6 mice off dox and age-matched WT/B6 mice off dox. The CA fields of tTA/B6 mice showed no significant atrophy at 8 or 24 weeks postweaning age compared with age-matched WT mice off dox (Fig. 13).
Volumetric MRI measurements in bigenic mice corroborate histological patterns of atrophy

Our histological findings were corroborated by in vivo quantitative MRI measures of the hippocampus (Fig. 14A) and the insula (Fig. 14B). Bigenic mice off dox showed significantly reduced volume of the hippocampus compared with bigenic mice on dox (mice on dox: $18.0 \pm 0.2 \mathrm{~mm}^{3}$, mice off dox: $17.3 \pm 0.3 \mathrm{~mm}^{3}$; two-way ANOVA; $F_{(1,12)}=21.389, p=$ 0.043; Fig. 14C). Furthermore, bigenic mice off dox also showed significant atrophy of the insular cortex compared with bigenic mice on dox diet (mice on dox: $5.54 \pm 0.11 \mathrm{~mm}^{3}$, 


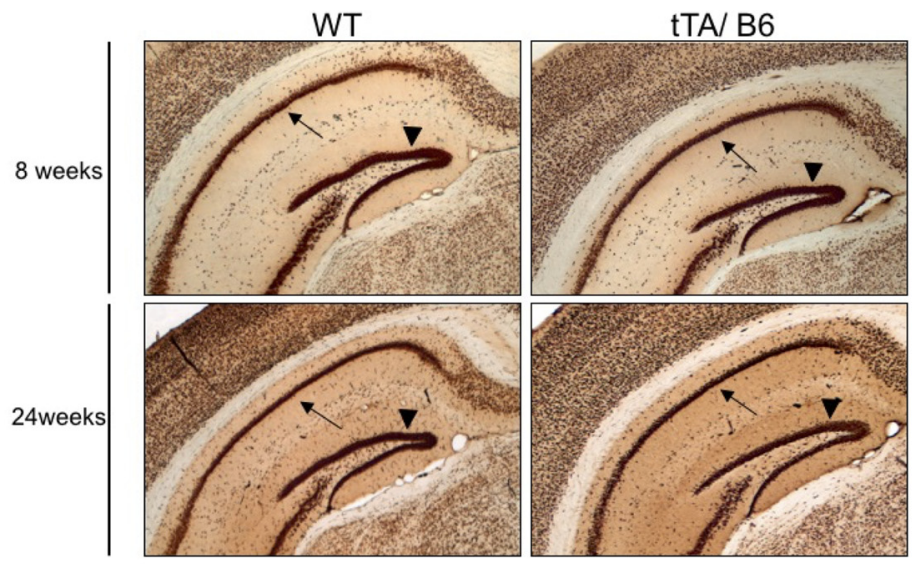

Dentate Gyrus

(8 weeks)

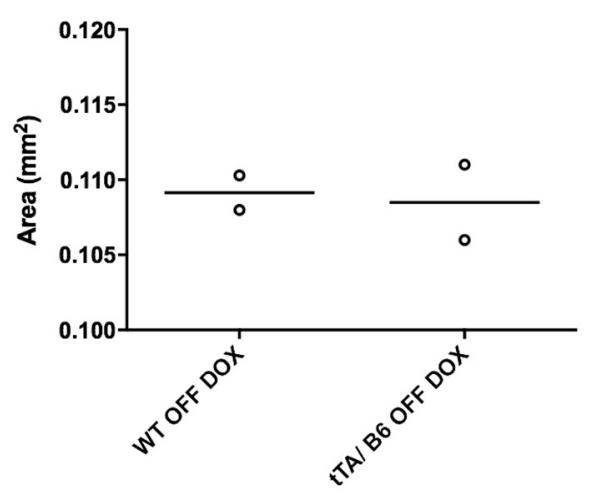

CA Fields of the Hippocampus (8 weeks)

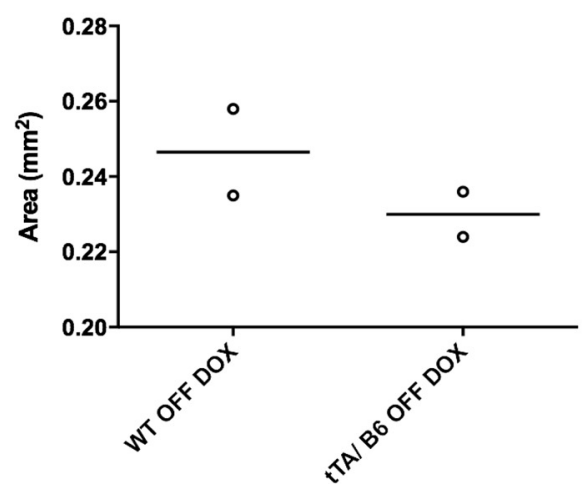

Dentate Gyrus

(24 weeks)

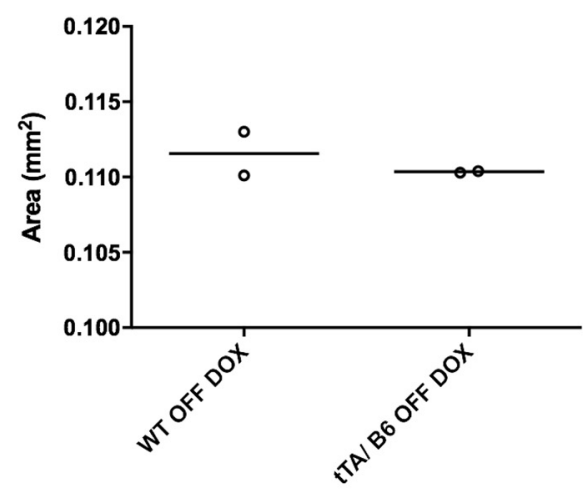

CA Fields of the Hippocampus (24 weeks)

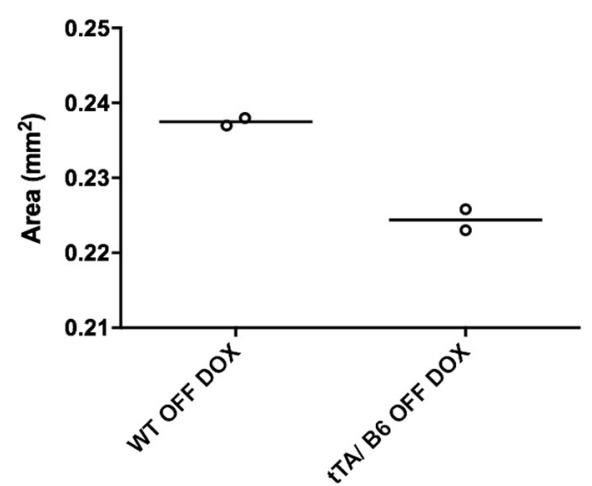

Figure 13. Mice on C57BL/6 background strain are rescued from tTA toxicity. Top, Single tTA transgenic mice that normally exhibit toxicity in the hippocampus on 129/SVE and FVB mix background (Figs. 3, 4) show no toxicity on C57BL/6 background strain expressing the tTA transgene (tTA/B6) off-doxycycline diet. Quantitative analysis shows that the mid-hippocampal DG (middle) and CA fields (bottom; represented in bregma $-2.54 \mathrm{~mm}$ ) are spared from atrophy in both 8 and 24 weeks postweaning age groups akin to phenotype in WT C57BL/6 mice $(n=2$ animals per genotype; circles represent individual data points; horizontal lines represent the mean).

mice off dox: $4.82 \pm 0.08 \mathrm{~mm}^{3}$; two-way ANOVA; $F_{(1,12)}=$ $5.548, p=0.001$; Fig. $14 D)$

\section{No significant changes in spatial memory due to tTA toxicity} Reference memory is associated with hippocampal function that can be tested using the Morris water maze (Lindner et al., 1992). Given that we observed significant neurodegeneration of the hippocampus in our bigenic animals due to tTA, we investigated whether this atrophy translated to memory impairment. Bigenic mice of 8 and 24 weeks postweaning age were tested over a $6 \mathrm{~d}$ period. There was no statistically significant difference in the interval to reach the platform on test days between mice on and off dox at 8 or 24 weeks postweaning age (Fig. 15). 

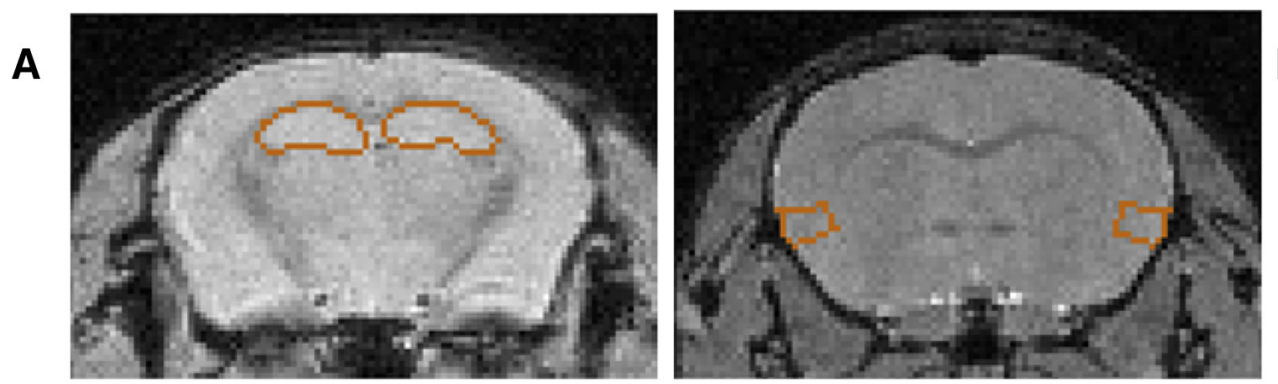

B

C

Hippocampus

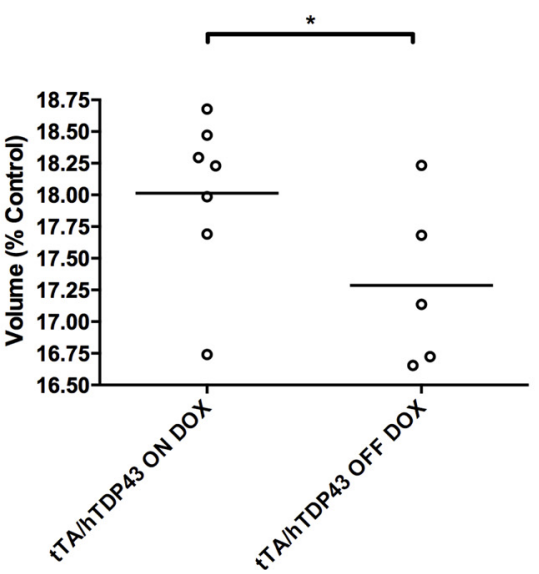

Insula

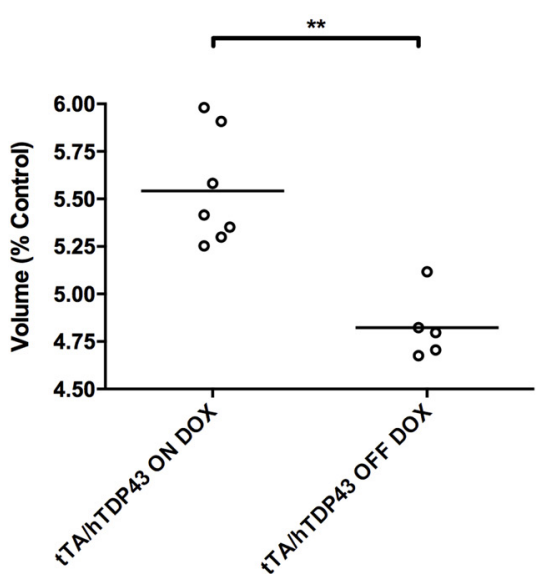

Figure 14. Quantitative MRI corroborates atrophy due to TTA transgene and TDP-43 overexpression. In vivo MR images of the $(\boldsymbol{A})$ hippocampus and $(\boldsymbol{B})$ insular cortex outlined in bigenic mice (tTA/hTDP-43) after 8 weeks of transgene expression. C, Bigenic mice off dox show significant atrophy of the hippocampus compared with age-matched bigenic mice on dox. D, Bigenic mice off dox also show significant atrophy in the insula compared with bigenic mice on dox. $n=5-7$ per genotype. Circles represent individual data points, horizontal lines represent the mean. ${ }^{*} p=0.043$, ${ }^{* *} p=0.001$.

\section{Discussion}

Tet-off expression systems are widely used to generate transgenic mice, which can conditionally express a gene of interest under the control of dox in their diet. In our mouse model, the expression system uses the tTA protein that drives overexpression of TDP43. Similar to previous findings, tTA caused phenotypes independent of those attributed to the transgene overexpression. We suspect that other mouse models that have also made use of the tet-off system potentially could have similar toxicity from the tTA expression (Yamasaki et al., 2007; Muyllaert et al., 2008; Schlatterer et al., 2011; Perez et al., 2013).

Expression of tTA can cause strain-dependent neuronal loss in the dentate granule cell layer of the hippocampus (Han et al., 2012). Our bigenic mice overexpressing TDP-43 using tTA as the driver exhibited similar patterns of toxicity in the hippocampus. We broadened our analysis to other subregions of the hippocampus (CA1-3). Hippocampal atrophy was detected at both 8 and 24 weeks postweaning age in the DG and CA fields. Atrophy was most severe in the mid-hippocampal region. This degeneration could be explained solely by expression of the tTA transgene, as single tTA transgenic mice showed almost the same pattern of atrophy. This suggests that toxicity in the hippocampus is relatively specific to overexpression of tTA, when the tetracycline response element is allowed to bind to tTA (off dox diet).

The neuronal loss in the DG was more conspicuous than degeneration in the CA fields. Hippocampal degeneration in the animals expressing tTA was apparent after 8 weeks postweaning age, and persisted at 24 weeks postweaning age. Previous studies corroborate our findings that tTA expression in transgenic mice is associated with small dentate gyri (Han et al., 2012; Liu et al., 2015). Genetic background was a major influence on degeneration induced by tTA. Our bigenic animals are a crossbreed of 129/SVE and FVB mouse strains, each of which has been shown to be susceptible to tTA-induced thinning of the dentate granule cell layer. Multiple mechanisms of strain-dependent toxicity could be at play that either change the level of tTA protein and/or the binding of tTA to its target DNA sites to confer susceptibility to or protection against tTA toxicity (Robertson et al., 2002). As previously reported, we found that dox treatment prevents neuronal loss in both the tTA single transgenic and bigenic animals, raising the possibility that dox provided in the animals' diet changes the conformation of the tTA protein, rendering it incapable of binding to TRE sequences leading to arrest of the degenerative phenotype. Han et al. (2012) were the first to show that backcrossing of CaMKIIa-tTA mice with the C57BL/6 (B6) mouse line results in protection against tTA-induced hippocampal degeneration. Using tTA single transgenic mice on B6 background, we examined the DG and CA fields compared with age-matched WT B6 mice. We found no significant toxicity of tTA in the hippocampus of tTA/ B6 mice at 8 or 24 weeks postweaning age compared with WT mice. Han et al. (2012) investigated potential protective mechanisms against tTA toxicity in B6 mice. They found that B6 mice carry a recessive allele, which acts as a protector against tTA-mediated physiological insults. Specifically, they identified a $12 \mathrm{Mbp}$ region on chromo- 
A

8 Weeks

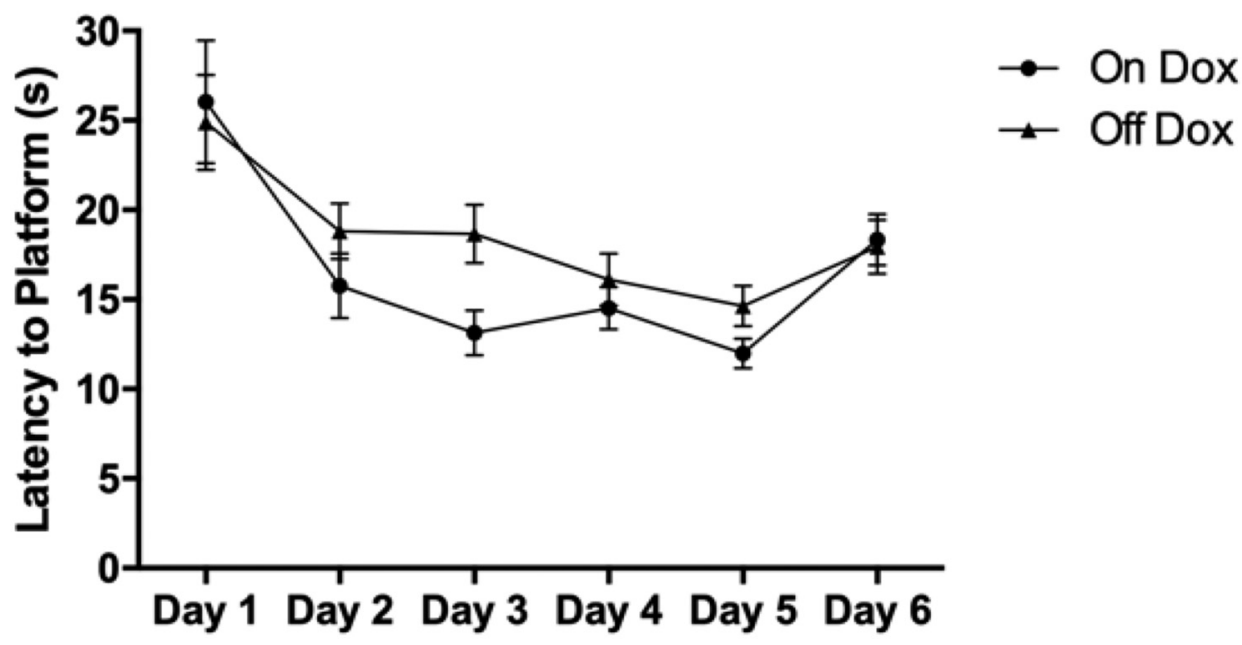

B
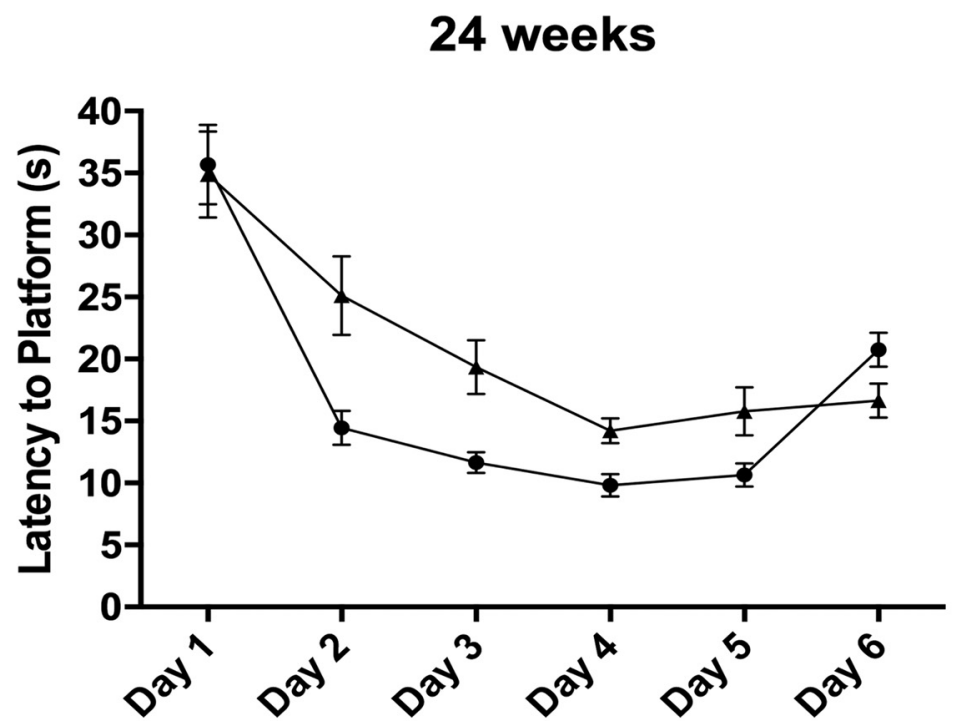

Figure 15. Hippocampal degeneration induced by tTA expression does not lead to loss of spatial memory. The Morris water maze was used to examine the effects of tTA-induced hippocampal degeneration on hippocampally-dependent spatial memory. Latency to reach platform does not vary significantly in bigenic mice off dox compared with bigenic mice on dox (controls) either at 8 $(\boldsymbol{A})$ or $24(\boldsymbol{B})$ weeks postweaning age $(n=16-21, p>0.05)$.

some 14, which was associated with dentate preservation in homozygous animals. This region of chromosome 14 is known to contain the clusterin gene (ApolipoproteinJ; Harold et al., 2009; Han et al., 2012). Studies investigating clusterin levels in another tTA-expressing mouse model report a 2.1-fold increase compared with WT mice (McCloskey et al., 2005). This finding suggests that tTA may interact with clusterin to produce hippocampal toxicity in transgenic mice.

In the original characterization of the tTA/hTDP-43 bigenic mice, Cannon et al. (2012) noted neurodegeneration in the DG. We conducted a more thorough examination of subregions of the hippocampus, and other cortical regions, which included the insula, piriform olfactory cortex, entorhinal cortex, cingulate cortex, prefrontal cortex, somatosensory cortex, and motor cortex. The degeneration in the hippocampus extended beyond the DG and into the CA fields primarily due to tTA expression. On the other hand, we detected significant atrophy in response to
TDP-43 overexpression in the insula, piriform cortex, entorhinal cortex, somatosensory cortex, and dorsolateral prefrontal cortex at 8 and/or 24 weeks postweaning age. But, there was no tTAspecific toxicity in these other cortical areas. The cingulate and motor cortices were spared from TDP-43-specific toxicity. In some cortical regions such as the dorsolateral prefrontal cortex, we observed more significant atrophy at 8 weeks postweaning age compared with 24 weeks. Although the mechanism for this agerelated recovery is currently unknown, one possible explanation may be that after the initial insult, there is neuronal atrophy (reduction in size), giving rise to overall cortical atrophy. If damage is not severe, neurons may be able to recover. However, if there is severe damage, the result is cell death as seen in the DG.

These observations suggest that tTA-induced toxicity is restricted to the hippocampus, and that neurodegeneration specifically due to hTDP-43 overexpression is selectively present in a number of limbic and neocortical regions. Thus, our tTA/ 
hTDP-43 bigenic mice represent a good model for investigation of the effects of hTDP-43 overexpression in non-hippocampal cortical regions. In addition, our hTDP-43 mouse model is unique among mouse models of FTLD disease where the expression and pathology of TDP-43 is targeted exclusively to the forebrain. In our animal model, regions such as the cerebellum and spinal cord (data not shown) were spared from TDP-43 pathology and associated atrophy.

Cortical atrophy in our animal model was accompanied by significant activation of microglia and astrocytes, consistent with neuronal damage. Atrophy appeared to be due to apoptotic cell death as evidenced by activation of caspase 3 and significant loss of NeuN-immunoreactive neurons.

There are other animal models of TDP-43 overexpression, but they recapitulate the TDP-43 pathology that underlies ALS/FTLD in which aggregates of TDP-43 accumulate in the spinal cord and motor neurons degenerate (Wegorzewska et al., 2009; Stallings et al., 2010; Igaz et al., 2011; Swarup et al., 2011; Esmaeili et al., 2013; Mitchell et al., 2015).

Future studies will be necessary to examine the hTDP-43 overexpressing mice in an environment independent of the toxicity from tTA, the driver of the transgene. In particular, these mice could be backcrossed to the B6 mouse background that has been shown to reduce or prevent tTA toxicity (Han et al., 2012).

Structural MRI is used extensively in clinical settings to detect patterns of atrophy characteristic of different neurodegenerative disorders (Frisoni et al., 2010; McEvoy and Brewer, 2010). To determine whether the atrophy we detected postmortem is also detectable in vivo, we subjected bigenic animals at 8 weeks postweaning age on or off dox to structural MRI analysis. The hippocampus, which displayed significant atrophy in both tTA single transgenic and bigenic animals in our histological studies, and the insula, which displayed significant atrophy only in bigenic mice, were analyzed. In both structures, we detected significant loss of volume in bigenic mice off dox compared with bigenic mice on dox, corroborating our histological findings, and indicating that structural MRI can be successfully used in transgenic mice for detection of atrophy patterns. However, study of such atrophy in bigenic animals will require further investigation to determine the atrophy due to expression of each transgene.

Behavioral testing conducted on bigenic mice revealed relatively intact reference memory despite significant atrophy of the hippocampus. This surprising finding suggests that the hippocampus can withstand relatively significant neurodegeneration without detectable functional deficits. The threshold beyond which such degeneration translates into behavioral deficits is at present unknown. It is also possible that damage to structures with significant connections to the hippocampus is necessary to cause memory deficits. Our findings have important implications for neurodegenerative disorders, such as Alzheimer's disease, in which loss of memory is a significant clinical feature.

In summary, the results of the present study provide a note of caution when using the tet-off system to generate transgenic animals because of the specific hippocampal toxicity due to tTA expression. However, in our bigenic animals, most cortical areas displayed atrophy that was not present in tTA single transgenic animals, indicating presence of atrophy uniquely due to TDP-43 overexpression. Significantly, some of the areas that displayed significant TDP-43-dependent degeneration, such as the dorsolateral prefrontal cortex, also display significant atrophy in FTLD with TDP-43 pathology. Thus, our bigenic animals appear to be appropriate for investigation of the role of TDP-43 pathology in non-hippocampal cortical areas.

\section{References}

Balconi M, Cotelli M, Brambilla M, Manenti R, Cosseddu M, Premi E, Gasparotti R, Zanetti O, Padovani A, Borroni B (2015) Understanding emotions in frontotemporal dementia: the explicit and implicit emotional cue mismatch. J Alzheimers Dis 46:211-225. CrossRef Medline

Barmada SJ, Skibinski G, Korb E, Rao EJ, Wu JY, Finkbeiner S (2010) Cytoplasmic mislocalization of TDP-43 is toxic to neurons and enhanced by a mutation associated with familial amyotrophic lateral sclerosis. J Neurosci 30:639-649. CrossRef Medline

Brettschneider J, Del Tredici K, Irwin DJ, Grossman M, Robinson JL, Toledo JB, Fang L, Van Deerlin VM, Ludolph AC, Lee VM, Braak H, Trojanowski JQ (2014) Sequential distribution of pTDP-43 pathology in behavioral variant frontotemporal dementia (bvFTD). Acta Neuropathol 127:423439. CrossRef Medline

Buratti E, De Conti L, Stuani C, Romano M, Baralle M, Baralle F (2010) Nuclear factor TDP-43 can affect selected microRNA levels. FEBS J 277: 2268-2281. CrossRef Medline

Cairns NJ, Bigio EH, Mackenzie IR, Neumann M, Lee VM, Hatanpaa KJ, White CL 3rd, Schneider JA, Grinberg LT, Halliday G, Duyckaerts C, Lowe JS, Holm IE, Tolnay M, Okamoto K, Yokoo H, Murayama S, Woulfe J, Munoz DG, Dickson DW, Ince PG, Trojanowski JQ, Mann DM (2007) Consortium for frontotemporal lobar degeneration neuropathologic diagnostic and nosologic criteria for frontotemporal lobar degeneration: consensus of the consortium for frontotemporal lobar degeneration. Acta Neuropathol 114:5-22. CrossRef Medline

Cannon A, Yang B, Knight J, Farnham IM, Zhang Y, Wuertzer CA, D'Alton S, Lin WL, Castanedes-Casey M, Rousseau L, Scott B, Jurasic M, Howard J, Yu X, Bailey R, Sarkisian MR, Dickson DW, Petrucelli L, Lewis J (2012) Neuronal sensitivity to TDP-43 overexpression is dependent on timing of induction. Acta Neuropathol 123:807-823. CrossRef Medline

Chen-Plotkin AS, Lee VM, Trojanowski JQ (2010) TAR DNA-binding protein 43 in neurodegenerative disease. Nat Rev Neurol 6:211-220. CrossRef Medline

Colombo E, Farina C (2016) Astrocytes: key regulators of neuroinflammation. Trends Immunol 37:608-620. CrossRef Medline

D’Alton S, Altshuler M, Cannon A, Dickson DW, Petrucelli L, Lewis J (2014) Divergent Phenotypes in Mutant TDP-43 Transgenic Mice Highlight Potential Confounds in TDP-43 Transgenic Modeling. PLoS ONE 9:e86513. CrossRef Medline

D'Amelio M, Sheng M, Cecconi F (2012) Caspase-3 in the central nervous system: beyond apoptosis. Trends Neurosci 35:700-709. CrossRef Medline

Esmaeili MA, Panahi M, Yadav S, Hennings L, Kiaei M (2013) Premature death of TDP-43 (A315T) transgenic mice due to gastrointestinal complications prior to development of full neurological symptoms of amyotrophic lateral sclerosis. Int J Exp Pathol 94:56-64. CrossRef Medline

Frisoni GB, Fox NC, Jack CR Jr, Scheltens P, Thompson PM (2010) The clinical use of structural MRI in Alzheimer disease. Nat Rev Neurol 6:6777. CrossRef Medline

Gossen M, Bujard H (1992) Tight control of gene expression in mammalian cells by tetracycline-responsive promoters. Proc Natl Acad Sci U S A 89: 5547-5551. CrossRef Medline

Han HJ, Allen CC, Buchovecky CM, Yetman MJ, Born HA, Marin MA, Rodgers SP, Song BJ, Lu HC, Justice MJ, Probst FJ, Jankowsky JL (2012) Strain background influences neurotoxicity and behavioral abnormalities in mice expressing the tetracycline transactivator. J Neurosci 32:1057410586. CrossRef Medline

Harold D, Abraham R, Hollingworth P, Sims R, Gerrish A, Hamshere ML, Pahwa JS, Moskvina V, Dowzell K, Williams A, Jones N, Thomas C, Stretton A, Morgan AR, Lovestone S, Powell J, Proitsi P, Lupton MK, Brayne C, Rubinsztein DC, et al. (2009) Genome-wide association study identifies variants at CLU and PICALM associated with Alzheimer's disease. Nat Genet 41:1088-1093. CrossRef Medline

Igaz LM, Kwong LK, Lee EB, Chen-Plotkin A, Swanson E, Unger T, Malunda J, Xu Y, Winton MJ, Trojanowski JQ, Lee VM (2011) Dysregulation of the ALS-associated gene TDP-43 leads to neuronal death and degeneration in mice. J Clin Invest 121:726-738. CrossRef Medline

Jiang H, van Zijl PC, Kim J, Pearlson GD, Mori S (2006) DtiStudio: resource program for diffusion tensor computation and fiber bundle tracking. Comput Methods Programs Biomed 81:106-116. CrossRef Medline

Josephs KA (2008) Frontotemporal dementia and related disorders: deciphering the enigma. Ann Neurol 64:4-14. CrossRef Medline 
Kwong LK, Neumann M, Sampathu DM, Lee VM, Trojanowski JQ (2007) TDP-43 proteinopathy: the neuropathology underlying major forms of sporadic and familial frontotemporal lobar degeneration and motor neuron disease. Acta Neuropathol 114:63-70. CrossRef Medline

Lee EB, Lee VM, Trojanowski JQ (2011) Gains or losses: molecular mechanisms of TDP43-mediated neurodegeneration. Nat Rev Neurosci 13:3850. CrossRef Medline

Lindner MD, Balch AH, VanderMaelen CP (1992) Short forms of the "reference-" and "working-memory" Morris water maze for assessing age-related deficits. Behav Neural Biol 58:94-102. CrossRef Medline

Liu P, Paulson JB, Forster CL, Shapiro SL, Ashe KH, Zahs KR (2015) Characterization of a novel mouse model of Alzheimer's disease: amyloid pathology and unique $\beta$-amyloid oligomer profile. PLoS One 10:e126317. CrossRef Medline

McCloskey DT, Turnbull L, Swigart PM, Zambon AC, Turcato S, Joho S, Grossman W, Conklin BR, Simpson PC, Baker AJ (2005) Cardiactransgenesis with the tetracycline transactivator changes myocardial function and gene expression. Physiol Genomics 22:118-126. CrossRef Medline

McEvoy LK, Brewer JB (2010) Quantitative structural MRI for early detection of Alzheimer's disease. Expert Rev Neurother 10:1675-1688. CrossRef Medline

Mitchell JC, Constable R, So E, Vance C, Scotter E, Glover L, Hortobagyi T, Arnold ES, Ling SC, McAlonis M, Da Cruz S, Polymenidou M, Tessarolo L, Cleveland DW, Shaw CE (2015) Wild type human TDP-43 potentiates ALS-linked mutant TDP-43 driven progressive motor and cortical neuron degeneration with pathological features of ALS. Acta Neuropathol Commun 3:36. CrossRef Medline

Morris RG, Garrud P, Rawlins JN, O’Keefe J (1982) Place navigation impaired in rats with hippocampal lesions. Nature 297:681-683. CrossRef Medline

Muyllaert D, Terwel D, Kremer A, Sennvik K, Borghgraef P, Devijver H, Dewachter I, Van Leuven F (2008) Neurodegeneration and neuroinflammation in cdk5/p25-inducible mice: a model for hippocampal sclerosis and neocortical degeneration. Am J Pathol 172:470-485. CrossRef Medline

Narayanan RK, Mangelsdorf M, Panwar A, Butler TJ, Noakes PG, Wallace RH (2013) Identification of RNA bound to the TDP-43 ribonucleoprotein complex in the adult mouse brain. Amyotroph Lateral Scler Frontotemporal Degener 14:252-260. CrossRef Medline

Paxinos G, Franklin KBJ (2001) The mouse brain in stereotaxic coordinates, Ed 2nd. San Diego, CA: Academic.

Perez PD, Hall G, Kimura T, Ren Y, Bailey RM, Lewis J, Febo M, Sahara N
(2013) In vivo functional brain mapping in a conditional mouse model of human tauopathy (tauP301L) reveals reduced neural activity in memory formation structures. Mol Neurodegener 8:9. CrossRef Medline

Perry VH, Holmes C (2014) Microglial priming in neurodegenerative disease. Nat Rev Neurol 10:217-224. CrossRef Medline

Piguet O, Hornberger M, Mioshi E, Hodges JR (2011) Behavioural-variant frontotemporal dementia: diagnosis, clinical staging, and management. Lancet Neurol 10:162-172. CrossRef Medline

Robertson A, Perea J, Tolmachova T, Thomas PK, Huxley C (2002) Effects of mouse strain, position of integration and tetracycline analogue on the tetracycline conditional system in transgenic mice. Gene 282:65-74. CrossRef Medline

Schlatterer SD, Tremblay MA, Acker CM, Davies P (2011) Neuronal cAbl overexpression leads to neuronal loss and neuroinflammation in the mouse forebrain. J Alzheimers Dis 25:119-133. CrossRef Medline

Stallings NR, Puttaparthi K, Luther CM, Burns DK, Elliott JL (2010) Progressive motor weakness in transgenic mice expressing human TDP-43. Neurobiol Dis 40:404-414. CrossRef Medline

Swarup V, Phaneuf D, Bareil C, Robertson J, Rouleau GA, Kriz J, Julien JP (2011) Pathological hallmarks of amyotrophic lateral sclerosis/frontotemporal lobar degeneration in transgenic mice produced with TDP-43 genomic fragments. Brain 134:2610-2626. CrossRef Medline

Tollervey JR, Curk T, Rogelj B, Briese M, Cereda M, Kayikci M, König J, Hortobágyi T, Nishimura AL, Zupunski V, Patani R, Chandran S, Rot G, Zupan B, Shaw CE, Ule J (2011) Characterizing the RNA targets and position-dependent splicing regulation by TDP-43. Nat Neurosci 14:452458. CrossRef Medline

Wegorzewska I, Bell S, Cairns NJ, Miller TM, Baloh RH (2009) TDP-43 mutant transgenic mice develop features of ALS and frontotemporal lobar degeneration. Proc Natl Acad Sci U S A 106:18809-18814. CrossRef Medline

Xu YF, Gendron TF, Zhang YJ, Lin WL, D’Alton S, Sheng H, Casey MC, Tong J, Knight J, Yu X, Rademakers R, Boylan K, Hutton M, McGowan E, Dickson DW, Lewis J, Petrucelli L (2010) Wild-type human TDP-43 expression causes TDP-43 phosphorylation, mitochondrial aggregation, motor deficits, and early mortality in transgenic mice. J Neurosci 30: 10851-10859. CrossRef Medline

Yamasaki TR, Blurton-Jones M, Morrissette DA, Kitazawa M, Oddo S, LaFerla FM (2007) Neural stem cells improve memory in an inducible mouse model of neuronal loss. J Neurosci 27:11925-11933. CrossRef Medline 